\title{
Unitarising Matrix Element + Parton Shower merging*
}

\author{
Leif Lönnblad and Stefan Prestel \\ Dept. of Astronomy and Theoretical Physics, Lund University, Sweden \\ E-mail: Leif.Lonnblad@thep.lu.se and Stefan.Prestel@thep.lu.se
}

\begin{abstract}
We revisit the CKKW-L method for merging tree-level matrix elements with parton showers, and amend it with an add/subtract scheme to minimise dependencies on the merging scale. The scheme is constructed to, as far as possible, recover the unitary nature of the underlying parton shower, so that the inclusive cross section is retained for each jet multiplicity separately.
\end{abstract}

Keywords: QCD, Jets, Parton Model, Phenomenological Models.

\footnotetext{
*Work supported in parts by the Swedish research council (contracts 621-2009-4076 and 621-2010-3326).
} 


\section{Contents}

1. Introduction 1

2. Parton shower unitarity 2

3. The problem with CKKW-L 6

4. Concepts of UMEPS 9

4.1 Procedure step-by-step 12

5. Results 14

5.1 W-boson production 15

5.2 Dijet production 20

5.3 Comparison with data 21

6. Discussion 25

7. Conclusions and Outlook 27

\section{Introduction}

Particularly after the discovery of a Higgs-candidate resonance at ATLAS and CMS, the precise description of multi-jet Standard Model (SM) processes at the LHC remains crucial. Major progress has recently been made in combining calculations of next-to-leading order (NLO) perturbative QCD corrections with Parton Shower (PS) based Monte Carlo event generators $[1-8]$.

In light of the rapid succession of publications on merging multiple NLO calculations with event generators $[1,6-8]$, it seems hardly arguable that this long-standing issue is resolved. From the point of next-to-leading order accuracy, scepticism about state-of-the-art methods is baseless. It has however also been pointed out that sub-leading logarithmic enhancements could be left after the merging procedure [9-11]. It has to be stressed that initially, this problem is not caused by the extension of tree-level methods to NLO, but already appears for CKKW-inspired tree-level merging schemes [12-16]. The introduction of a merging scale $\left(t_{\mathrm{MS}}\right)$ introduces logarithmic dependencies, $L=\ln \mu_{F} / t_{\mathrm{MS}}$, with a dominant contribution $\alpha_{\mathrm{s}}^{n} L^{2 n}$, in the multi-jet tree-level configurations. These terms are partly cancelled by the parton shower higher-order corrections to low-multiplicity states. Let us look at one-jet merging in W-boson production, with a strictly leading-logarithmic parton shower. Integrating the $\mathrm{W}+$ jet matrix element $(\mathrm{ME})$ over the one-jet phase space introduces the dependencies $\alpha_{\mathrm{s}} L^{2}$ and $\alpha_{\mathrm{s}} L^{1}$, while unresolved PS emissions in the zero-jet 
state produce $\alpha_{\mathrm{s}} L^{2}$ terms, but with negative sign. In the total cross section, the $\alpha_{\mathrm{s}} L^{2}$ terms cancel, and only a $t_{\mathrm{MS}}$-dependence beyond the accuracy of the parton shower (i.e. $\alpha_{\mathrm{s}} L^{1}$-terms) remains. This example is rather academic, since modern parton showers include a matrix-element correction for $\mathrm{W}+$ jet production, thus ensuring that in one-jet merging, all dependencies on $t_{\mathrm{MS}}$ are cancelled ${ }^{1}$. After the inclusion of matrix element corrections [17-20], the merging scale dependence enters for two-jet merging. However, since control of beyond-leading logarithmic contributions cannot be universally exerted in a parton shower, it is clear that dependencies enter at some level, commonly hoped to be at next-to-next-to-leading logarithmic accuracy. The logarithmic structure of a two-jet tree-level cross section for example has only an approximate equivalent in parton showers, meaning that certainly at $\mathcal{O}\left(\alpha_{\mathrm{s}}^{2}\right)$, sub-leading contributions are not fully cancelled. This is often, for lack of a better term, referred to as violation of PS unitarity.

We believe it important to investigate this issue more closely. In this publication, we take a step back from the remarkable progress in NLO merging and outline a tree-level merging method that amends the behaviour of parton showers to ensure that no spurious logarithmic enhancements are introduced by including multi-jet matrix elements. The foundation of this method is PS unitarity, i.e. the requirement that the lowest order cross section remains unchanged by methods introduced to ameliorate the description of shapes of observable. It should be noted that in particular the GKS matrix-element-correction method in VINCIA [21,22] has emphasised a unitarity-based approach before. The aim of this article is more modest, in that we offer a novel prescription of combining the input used in multi-jet merging procedures, moving from the additive scheme of CKKW-inspired methods to an add-and-subtract method that preserves the total inclusive cross section.

This article is structured as follows. Section 2 is intended as introduction to our perspective on PS unitarity. This will be succeeded by a brief discussion of tree-level merging in the CKKW-L scheme $[13,14,16]$ and its problems in section 3, before we move on to construct a LO merging method that preserves PS unitarity in section 1 . Section 5 presents results for including additional jets in W-production and QCD dijet processes in the novel procedure, which we call UMEPS (Unitary Matrix Element + Parton Shower merging). Finally, we give a discussion in section 6 and conclude in section 7 .

\section{Parton shower unitarity}

Without any outside intervention, parton showers act on a lowest order seed cross section as a unitary operator. In other words, showering dresses the constituents of a perturbatively calculated $2 \rightarrow 2$ process with radiation, in order to set the stage for hadronisation. By generating soft and collinear emissions, parton showering sums (at least) leading doublelogarithmic enhancements to all orders.

Before detailing how this is achieved, let us introduce some notation in order to make the formulae less cluttered. We will also classify partons to be either resolved jets or unresolved jets at a particular scale $\rho_{\mathrm{MS}}$. By this, we mean that in a jet algorithm that

\footnotetext{
${ }^{1}$ Note that the introduction of ME corrections already endows the PS with the tree-level description of the hardest jet, so that one-jet merging would not be necessary.
} 
exactly inverts the parton shower, a parton would be resolved as a jet if the evolution scale at which it was emitted $(\rho)$, as well as jet separations that have been changed by the emission due to recoil effects, are above $\rho_{\mathrm{MS}}$. For the parton shower to be invertible in a well-defined way, we assume the existence of on-shell intermediate states between splittings. The index MS foreshadows the use of this jet definition as merging scale ${ }^{2}$.

The parton shower approximates the effect of virtual corrections on observables sensitive to the scales $\rho_{i}$ and $\rho_{i+1}$ by integrating DGLAP splitting kernels $P(z)$ over the unresolved phase space. This gives a term

$$
\begin{gathered}
-\int_{\rho_{i+1}}^{\rho_{i}} d \rho d z \frac{\alpha_{\mathrm{S}}(\rho)}{2 \pi}\left\{\sum_{a \in\{\text { outgoing }\}} \sum_{j} P_{j}^{a}(z)+\sum_{a \in\{\text { incoming }\}} \sum_{j} \frac{f_{j}^{a}\left(\frac{x_{i}^{a}}{z}, \rho\right)}{f_{i}^{a}\left(x_{i}^{a}, \rho\right)} P_{j}^{a}(z)\right\} \\
\equiv-\int_{\rho_{i+1}}^{\rho_{i}} d \rho d z \alpha_{\mathrm{S}}(\rho) \mathcal{P}_{i+1}(z, \rho),
\end{gathered}
$$

where the first terms on the left-hand side sums all possible unresolved final state emissions, and the second term includes all unresolved initial state splittings. The ratios of parton distribution functions $f$ is only absent if no initial parton is taking part in the (unresolved) emissions. The $\mathcal{P}_{i+1}$ (and $P$ ) notation is rather symbolic to permit a certain degree of simplicity. We include symmetry factors and the typical $\rho$ - and $z$-fractions from approximating the matrix element or multiplying Jacobian factors in $P$ and $\mathcal{P}_{i+1}$, e.g. for an initial state splitting $\overline{\mathrm{q}} \rightarrow \overline{\mathrm{q}} \mathrm{g}$, we would have $P=\frac{1}{\rho} \frac{1}{z} \frac{1+z^{2}}{1-z}$. It can be shown, by performing the $z$-integration for a specific splitting kernel $P$, that the PS "virtual corrections" in eq. (2.1) indeed capture the leading logarithmic contributions of virtual corrections. Let us introduce the short-hand

$$
f_{i}\left(x_{i}, \rho_{i}\right)=f_{i}^{+}\left(x_{i}^{+}, \rho_{i}\right) f_{i}^{-}\left(x_{i}^{-}, \rho_{i}\right)
$$

The Sudakov form factor, resumming unresolved emissions between scales $\rho_{i}$ and $\rho_{i+1}$, is given by

$$
\Delta_{\mathrm{S}_{+\mathrm{i}}}\left(x_{i}, \rho_{i}, \rho_{i+1}\right)=\frac{f_{i}\left(x_{i}, \rho_{i}\right)}{f_{i+1}\left(x_{i}, \rho_{i+1}\right)} \Pi_{S_{+i}}\left(x_{i}, \rho_{i}, \rho_{i+1}\right)
$$

where $\Pi_{S_{+i}}\left(x_{i}, \rho_{i}, \rho_{i+1}\right)$ is the probability of no emission from state $S_{+i}$ between the $\rho_{i}$ and $\rho_{i+1}$. The no-emission probability in turn can be expressed as

$$
\Pi_{S_{+i}}\left(x_{i}, \rho_{i}, \rho_{i+1}\right)=\exp \left\{-\int_{\rho_{i+1}}^{\rho_{i}} d \rho d z \alpha_{\mathrm{s}}(\rho) \mathcal{P}_{i+1}(z, \rho)\right\}
$$

We have kept $x_{i}$ as an argument to remember that $\Pi_{S_{+i}}\left(x_{i}, \rho_{i}, \rho_{i+1}\right)$ contains $x_{i}$-dependent PDF factors through $\mathcal{P}_{i+1}$.

\footnotetext{
${ }^{2}$ We here restrict ourselves to a particular jet (merging scale) definition, for the sake of clarity. We further assumed that the PS evolution variable is a measure of "hardness", i.e. that soft and collinear divergences are located at $\rho \rightarrow 0$. All the following arguments apply for a general merging scale $t_{\mathrm{MS}}$.
} 
Let us consider the case when no PS emission above a scale $\rho_{\mathrm{MS}}$ is generated. The parton shower approximation of the resummed exclusive zero-jet cross section is then

$$
\begin{aligned}
\frac{d \sigma_{0}^{e x}}{d \phi_{0}} & =f_{0}\left(x_{0}, \rho_{0}\right)\left|\mathcal{M}_{0}\left(\rho_{0}, \mu_{R}\right)\right|^{2} \Pi_{S_{+0}}\left(x_{0}, \rho_{0}, \rho_{\mathrm{MS}}\right) \\
& =f_{0}\left(x_{0}, \rho_{0}\right)\left|\mathcal{M}_{0}\left(\rho_{0}, \mu_{R}\right)\right|^{2} \\
& -f_{0}\left(x_{0}, \rho_{0}\right)\left|\mathcal{M}_{0}\left(\rho_{0}, \mu_{R}\right)\right|^{2} \int_{\rho_{\mathrm{MS}}}^{\rho_{0}} d \rho d z \alpha_{\mathrm{S}}(\rho) \mathcal{P}_{1}(z, \rho) \\
& +f_{0}\left(x_{0}, \rho_{0}\right)\left|\mathcal{M}_{0}\left(\rho_{0}, \mu_{R}\right)\right|^{2} \int_{\rho_{\mathrm{MS}}}^{\rho_{0}} d \rho_{1} d z_{1} \alpha_{\mathrm{S}}\left(\rho_{1}\right) \mathcal{P}_{1}\left(z_{1}, \rho_{1}\right) \\
& \times \int_{\rho_{\mathrm{MS}}}^{\rho_{1}} d \rho_{2} d z_{2} \alpha_{\mathrm{S}}\left(\rho_{2}\right) \mathcal{P}_{1}\left(z_{2}, \rho_{2}\right)+\mathcal{O}\left(\alpha_{\mathrm{s}}^{3}\right),
\end{aligned}
$$

where we have used the fact that

$$
\begin{aligned}
& \frac{1}{2}\left(\int_{\rho_{\mathrm{MS}}}^{\rho_{0}} d \rho d z \alpha_{\mathrm{S}}(\rho) \mathcal{P}_{1}(z, \rho)\right)^{2} \\
& =\int_{\rho_{\mathrm{MS}}}^{\rho_{0}} d \rho_{1} d z_{1} \alpha_{\mathrm{S}}\left(\rho_{1}\right) \mathcal{P}_{1}\left(z_{1}, \rho_{1}\right) \int_{\rho_{\mathrm{MS}}}^{\rho_{1}} d \rho_{2} d z_{2} \alpha_{\mathrm{S}}\left(\rho_{2}\right) \mathcal{P}_{1}\left(z_{2}, \rho_{2}\right) .
\end{aligned}
$$

The zero-jet PS cross section is exclusive in the sense that no resolved emissions (i.e. emissions above $\rho_{\mathrm{MS}}$ ) are produced. Beside resumming unresolved contributions to the zero-jet cross section, the parton shower also produces resolved emissions. The parton shower approximation to the cross section for emitting the hardest jet at scale $\rho_{1}$ is

$$
\frac{d \sigma_{1}^{i n}}{d \phi_{0}}=d \rho_{1} d z_{1} f_{0}\left(x_{0}, \rho_{0}\right)\left|\mathcal{M}_{0}\left(\rho_{0}, \mu_{R}\right)\right|^{2} \alpha_{\mathrm{s}}\left(\rho_{1}\right) \mathcal{P}_{1}\left(z_{1}, \rho_{1}\right) \Pi_{S_{+0}}\left(x_{0}, \rho_{0}, \rho_{1}\right)
$$

$\mathcal{P}_{1}$ contains PDF ratios and kinematical factors (see definition 2.1). The cross section is labelled with in for "inclusive", because the emission of further jets below $\rho_{1}$, but above $\rho_{\mathrm{MS}}$, is still allowed. Note that $\frac{d \sigma_{1}^{i n}}{d \phi_{0}}$ is also exclusive in the sense that no resolved emissions above $\rho_{1}$ - the scale of the first emission - are possible. In the following, we will always call a cross section inclusive if the parton shower can (at least in principle) produce further resolved emissions, and exclusive otherwise.

If only zero- and one-jet states are generated, the total cross section is given by the sum of exclusive zero-jet and inclusive one-jet cross sections. Let us analyse the total cross section in the approximation of having maximally one parton shower emission. It useful to rewrite eq. (2.5) with help of definition eq. (2.4):

$$
\begin{aligned}
\frac{d \sigma_{0}^{e x}}{d \phi_{0}} & =f_{0}\left(x_{0}, \rho_{0}\right)\left|\mathcal{M}_{0}\left(\rho_{0}, \mu_{R}\right)\right|^{2} \\
& -f_{0}\left(x_{0}, \rho_{0}\right)\left|\mathcal{M}_{0}\left(\rho_{0}, \mu_{R}\right)\right|^{2} \int_{\rho_{\mathrm{MS}}}^{\rho_{0}} d \rho d z \alpha_{\mathrm{S}}(\rho) \mathcal{P}_{1}(z, \rho) \Pi_{S_{+0}}\left(x, \rho_{0}, \rho\right)
\end{aligned}
$$


so that the total cross section is

$$
\begin{aligned}
\sigma^{i n}= & \int d \phi_{0}\left(\frac{d \sigma_{0}^{e x}}{d \phi_{0}}+\int \frac{d \sigma_{1}^{i n}}{d \phi_{0}}\right) \\
= & \int d \phi_{0}\left(f_{0}\left(x_{0}, \rho_{0}\right)\left|\mathcal{M}_{0}\left(\rho_{0}, \mu_{R}\right)\right|^{2}\right. \\
& \left.\quad-f_{0}\left(x_{0}, \rho_{0}\right)\left|\mathcal{M}_{0}\left(\rho_{0}, \mu_{R}\right)\right|^{2} \int_{\rho_{\mathrm{MS}}}^{\rho_{0}} d \rho d z \alpha_{\mathrm{S}}(\rho) \mathcal{P}_{1}(z, \rho) \Pi_{S_{+0}}\left(x, \rho_{0}, \rho\right)\right) \\
+ & \int d \phi_{0} f_{0}\left(x_{0}, \rho_{0}\right)\left|\mathcal{M}_{0}\left(\rho_{0}, \mu_{R}\right)\right|^{2} \int_{\rho_{\mathrm{MS}}}^{\rho_{0}} d \rho d z \alpha_{\mathrm{s}}(\rho) \mathcal{P}(z, \rho) \Pi_{S_{+0}}\left(x_{0}, \rho_{0}, \rho\right) \\
= & \int d \phi_{0} f_{0}\left(x_{0}, \rho_{0}\right)\left|\mathcal{M}_{0}\left(\rho_{0}, \mu_{R}\right)\right|^{2}
\end{aligned}
$$

Thus, if the parton shower would stop after the first emission, the total cross section is not changed by the application of the parton shower.

This small sketch does not really ensure that the total cross section is preserved after PS resummation. Parton showering usually generates more than one emission, so that only being concerned with a single emission might not be enough. The above argument can however be extended to any number of emissions. As an example, assume the PS had generated two emissions. Then, the one-jet cross section becomes exclusive by demanding that only one resolved emission has been produced,

$$
\begin{aligned}
\frac{d \sigma_{1}^{e x}}{d \phi_{0}}= & d \rho_{1} d z_{1} f_{0}\left(x_{0}, \rho_{0}\right)\left|\mathcal{M}_{0}\left(\rho_{0}, \mu_{R}\right)\right|^{2} \\
& \alpha_{\mathrm{s}}\left(\rho_{1}\right) \mathcal{P}_{1}\left(z_{1}, \rho_{1}\right) \Pi_{S_{+0}}\left(x_{0}, \rho_{0}, \rho_{1}\right) \Pi_{S_{+1}}\left(x_{1}, \rho_{1}, \rho_{\mathrm{MS}}\right)
\end{aligned}
$$

and we need to add the PS approximation to the two-jet cross section

$$
\begin{aligned}
\frac{d \sigma_{2}^{i n}}{d \phi_{0}}= & d \rho_{1} d z_{1} d \rho_{2} d z_{2} f_{0}\left(x_{0}, \rho_{0}\right)\left|\mathcal{M}_{0}\left(\rho_{0}, \mu_{R}\right)\right|^{2} \\
& \times \alpha_{\mathrm{s}}\left(\rho_{1}\right) \mathcal{P}_{1}\left(z_{1}, \rho_{1}\right) \Pi_{S_{+0}}\left(x_{0}, \rho_{0}, \rho_{1}\right) \\
& \times \alpha_{\mathrm{s}}\left(\rho_{2}\right) \mathcal{P}_{2}\left(z_{2}, \rho_{2}\right) \Pi_{S_{+1}}\left(x_{1}, \rho_{1}, \rho_{2}\right) \Theta\left(\rho_{1}-\rho_{2}\right)
\end{aligned}
$$

Now we rewrite 2.9 by expanding the second no-emission probability

$$
\begin{aligned}
\frac{d \sigma_{1}^{e x}}{d \phi_{0}}= & d \rho_{1} d z_{1} f_{0}\left(x_{0}, \rho_{0}\right)\left|\mathcal{M}_{0}\left(\rho_{0}, \mu_{R}\right)\right|^{2} \alpha_{\mathrm{S}}\left(\rho_{1}\right) \mathcal{P}_{1}\left(z_{1}, \rho_{1}\right) \Pi_{S_{+0}}\left(x_{0}, \rho_{0}, \rho_{1}\right) \\
& \times\left(1-\int_{\rho_{\mathrm{MS}}}^{\rho_{1}} d \rho_{2} d z_{2} \alpha_{\mathrm{S}}\left(\rho_{2}\right) \mathcal{P}_{2}\left(z_{2}, \rho_{2}\right)\right. \\
& \left.\quad+\int_{\rho_{\mathrm{MS}}}^{\rho_{1}} d \rho_{2} d z_{2} \int_{\rho_{\mathrm{MS}}}^{\rho_{2}} d \rho_{3} d z_{3} \alpha_{\mathrm{S}}\left(\rho_{3}\right) \mathcal{P}_{2}\left(z_{3}, \rho_{3}\right)+\mathcal{O}\left(\alpha_{\mathrm{s}}^{3}\right)\right) \\
= & d \rho_{1} d z_{1} f_{0}\left(x_{0}, \rho_{0}\right)\left|\mathcal{M}_{0}\left(\rho_{0}, \mu_{R}\right)\right|^{2} \alpha_{\mathrm{S}}\left(\rho_{1}\right) \mathcal{P}_{1}\left(z_{1}, \rho_{1}\right) \Pi_{S_{+0}}\left(x_{0}, \rho_{0}, \rho_{1}\right) \\
& \times\left(1-\int_{\rho_{\mathrm{MS}}}^{\rho_{1}} d \rho d z \alpha_{\mathrm{S}}(\rho) \mathcal{P}_{2}(z, \rho) \Pi_{S_{+1}}\left(x_{1}, \rho_{1}, \rho\right)\right)
\end{aligned}
$$


where we have again used definition 2.4 to derive the last equality. If parton showering stops after generating maximally two emissions, the total cross section is given by

$$
\sigma^{i n}=\int d \phi_{0}\left(\frac{d \sigma_{0}^{e x}}{d \phi_{0}}+\int \frac{d \sigma_{1}^{e x}}{d \phi_{0}}+\iint \frac{d \sigma_{2}^{i n}}{d \phi_{0}}\right)
$$

By comparing the second term in eq. (2.11) with eq. (2.10), we see that any PS contribution of two resolved jets cancels with terms containing one resolved and one unresolved jet. Already earlier, we saw that contributions with one resolved jet cancel against terms with zero resolved jets. Thus, we again find

$$
\sigma^{i n}=\int d \phi_{0} f_{0}\left(x_{0}, \rho_{0}\right)\left|\mathcal{M}_{0}\left(\rho_{0}, \mu_{R}\right)\right|^{2}
$$

It is easy to extend this argument to as many emissions as wanted: Whenever the parton shower generates one emission, the change in the total cross section is counteracted exactly by unresolved contributions to states with one emission less. There is no need to correct the PS approximation of the zero-jet exclusive cross section in the presence of two-jet states - the zero-jet resummation is oblivious of two-jet states. That parton showers are unitary is understandable directly from their construction, since the branching of an underlying $n$-jet state produces a $n+1$-jet state, which overwrites (i.e. removes) the $n$-jet state in the wake of the branching.

\section{The problem with CKKW-L}

Let us now review tree-level matrix element merging, more specifically the CKKW-L scheme $^{3}$. We here focus mainly on issues related to parton shower unitarity, and refer to [16] for a detailed description of CKKW-L in PYTHIA8 [23] and to [12-15] for a more general introduction.

Matrix element merging procedures are designed to improve the PS description of multi-jet observables. For this purpose, tree-level matrix element (ME) calculations are combined with the parton shower, i.e. tree-level-weighted phase space points with $m$ "hard process particles" and $n$ additional partons are included in the shower. In the following, we will often use the terms state, event, configuration or the symbol $S_{+n}$ to refer to these $n+m$-body phase space points. As can be inferred from the form of $S_{+n}$, we will most often understand that the state contains $m$ hard process particles, but not mention these particles explicitly.

A consistent merging removes all overlap between ME states and the PS approximation. This is ensured by introducing a phase space cut $\rho_{\mathrm{MS}}$ to separate the ME region from the PS region, and applying no-emission probabilities. The cut dependence is minimised by weighting configurations above and below $\rho_{\mathrm{MS}}$ in identical fashion. The CKKW-L scheme constructs and chooses a sequence of lower-multiplicity states (a so-called parton shower history) for each ME event, since factors need to be generated that would, in the parton

\footnotetext{
${ }^{3}$ Although most of what we discuss also applies to other CKKW-inspired merging schemes.
} 
shower evolution, have contributed though intermediate stages. With the help of the history, ME events will be reweighted with

$$
\begin{aligned}
w_{n}= & \frac{x_{0} f_{0}\left(x_{0}, \rho_{0}\right)}{x_{n} f_{n}\left(x_{n}, \mu_{F}\right)} \times\left(\prod_{i=1}^{n} \frac{x_{i} f_{i}\left(x_{i}, \rho_{i}\right)}{x_{i-1} f_{i-1}\left(x_{i-1}, \rho_{i}\right)}\right) \\
& \times\left(\prod_{i=1}^{n} \frac{\alpha_{\mathrm{s}}\left(\rho_{i}\right)}{\alpha_{\mathrm{s}}\left(\mu_{R}\right)}\right) \times\left(\prod_{i=1}^{n} \Pi_{S_{+i-1}}\left(\rho_{i-1}, \rho_{i}\right)\right) \times \Pi_{S_{+n}}\left(\rho_{n}, \rho_{\mathrm{MS}}\right) \\
= & \frac{x_{n} f_{n}\left(x_{n}, \rho_{n}\right)}{x_{n} f_{n}\left(x_{n}, \mu_{F}\right)} \times \prod_{i=1}^{n}\left[\frac{\alpha_{\mathrm{S}}\left(\rho_{i}\right)}{\alpha_{\mathrm{SME}}} \frac{x_{i-1} f_{i-1}\left(x_{i-1}, \rho_{i-1}\right)}{x_{i-1} f_{i-1}\left(x_{i-1}, \rho_{i}\right)} \Pi_{S_{+i-1}}\left(x_{i-1}, \rho_{i-1}, \rho_{i}\right)\right] \\
& \times \Pi_{S_{+n}}\left(x_{n}, \rho_{n}, \rho_{\mathrm{MS}}\right),
\end{aligned}
$$

where $\rho_{i}$ are the reconstructed splitting scales, and $S_{+i}$ the reconstructed intermediate states. The first PDF ratio in eq. (3.1) ensures that all ME configurations are normalised to the same total cross section, given by the lowest order Born-level matrix element. The PDF ratios in brackets account for PDF factors in the shower splitting probabilities $\mathcal{P}_{i}$ for initial state backward evolution. The running of $\alpha_{\mathrm{s}}$ is correctly included by the second bracket. Finally, double-counting is prevented by multiplying no-emission probabilities.

Let us investigate how the CKKW-L merging prescription changes the lowest-order inclusive cross section. For simplicity, we will highlight merging matrix elements with up to two additional jets with parton showers. In the simplest conceivable case of one-jet merging, applying CKKW-L defines the cross sections

$$
\begin{aligned}
\frac{d \sigma_{0}^{e x}}{d \phi_{0}} & =f_{0}\left(x_{0}, \rho_{0}\right)\left|\mathcal{M}_{0}\left(\rho_{0}, \mu_{R}\right)\right|^{2} \Pi_{S_{+0}}\left(x_{0}, \rho_{0}, \rho_{\mathrm{MS}}\right) \\
& =f_{0}\left(x_{0}, \rho_{0}\right)\left|\mathcal{M}_{0}\left(\rho_{0}, \mu_{R}\right)\right|^{2} \times\left(1-\int_{\rho_{\mathrm{MS}}}^{\rho_{0}} d \rho d z \alpha_{\mathrm{S}}(\rho) \mathcal{P}_{1}(z, \rho)+\mathcal{O}\left(\alpha_{\mathrm{s}}^{2}\right)\right) \\
\frac{d \sigma_{1}^{i n}}{d \phi_{0}} & =d \rho_{1} d z_{1} f_{0}\left(x_{0}, \rho_{0}\right) \frac{\alpha_{\mathrm{S}}\left(\rho_{1}\right)}{\alpha_{\mathrm{s}}\left(\mu_{R}\right)} \frac{f_{1}\left(x_{1}, \rho_{1}\right)}{f_{0}\left(x_{0}, \rho_{1}\right)}\left|\mathcal{M}_{1}\left(\rho_{0}, \mu_{R}\right)\right|^{2} \Pi_{S_{+0}}\left(x_{0}, \rho_{0}, \rho_{1}\right)
\end{aligned}
$$

It is crucial to note that the tree-level one-jet matrix element is in general different from the approximate PS splitting kernels. The inclusive lowest-order cross section is only preserved if

$$
\begin{array}{r}
f_{0}\left(x_{0}, \rho_{0}\right)\left|\mathcal{M}_{0}\left(\rho_{0}, \mu_{R}\right)\right|^{2} \int_{\rho_{\mathrm{MS}}}^{\rho_{0}} d \rho d z \alpha_{\mathrm{S}}(\rho) \mathcal{P}_{1}(z, \rho) \\
=\int_{\rho_{\mathrm{MS}}}^{\rho_{0}} d \rho_{1} d z_{1} f_{0}\left(x_{0}, \rho_{0}\right) \frac{\alpha_{\mathrm{S}}\left(\rho_{1}\right)}{\alpha_{\mathrm{s}}\left(\mu_{R}\right)} \frac{f_{1}\left(x_{1}, \rho_{1}\right)}{f_{0}\left(x_{0}, \rho_{1}\right)}\left|\mathcal{M}_{1}\left(\rho_{0}, \mu_{R}\right)\right|^{2}
\end{array}
$$

i.e. in the case where the first parton shower emission is distributed exactly according the one-jet matrix element. In this case, we would not have needed a merging prescription, since the PS would have already produced the correct result.

Though correcting the first PS splitting to the full tree-level result is reasonably simple, correcting higher multiplicities requires significantly more work. The VINCIA program aims at solving this issue $[21,22]$. In general however, we are currently forced to rely on tree-level merging to improve the descriptions of multi-jet observables. 
If a first-splitting-corrected PS is available, unitarity violations will enter when including matrix elements for the next higher jet multiplicity. Since the case of two-jet merging is also instructive for later considerations, we will list the contributions to the cross section below.

$$
\begin{aligned}
\frac{d \sigma_{0}^{e x}}{d \phi_{0}}= & f_{0}\left(x_{0}, \rho_{0}\right)\left|\mathcal{M}_{0}\left(\rho_{0}, \mu_{R}\right)\right|^{2} \Pi_{S_{+0}}\left(x_{0}, \rho_{0}, \rho_{\mathrm{MS}}\right) \\
= & f_{0}\left(x_{0}, \rho_{0}\right)\left|\mathcal{M}_{0}\left(\rho_{0}, \mu_{R}\right)\right|^{2} \\
& \times\left(1-\int_{\rho_{\mathrm{MS}}}^{\rho_{0}} d \rho d z \alpha_{\mathrm{S}}(\rho) \mathcal{P}_{1}(z, \rho) \Pi_{S_{+0}}\left(x_{0}, \rho_{0}, \rho\right)\right) \\
\frac{d \sigma_{1}^{e x}}{d \phi_{0}}= & d \rho_{1} d z_{1} f_{0}\left(x_{0}, \rho_{0}\right) \frac{\alpha_{\mathrm{s}}\left(\rho_{1}\right)}{\alpha_{\mathrm{s}}\left(\mu_{R}\right)} \frac{f_{1}\left(x_{1}, \rho_{1}\right)}{f_{0}\left(x_{0}, \rho_{1}\right)}\left|\mathcal{M}_{1}\left(\rho_{0}, \mu_{R}\right)\right|^{2} \\
& \Pi_{S_{+0}}\left(x_{0}, \rho_{0}, \rho_{1}\right) \Pi_{S_{+1}}\left(x_{1}, \rho_{1}, \rho_{\mathrm{MS}}\right) \\
= & d \rho_{1} d z_{1} f_{0}\left(x_{0}, \rho_{0}\right) \frac{\alpha_{\mathrm{S}}\left(\rho_{1}\right)}{\alpha_{\mathrm{S}}\left(\mu_{R}\right)} \frac{f_{1}\left(x_{1}, \rho_{1}\right)}{f_{0}\left(x_{0}, \rho_{1}\right)}\left|\mathcal{M}_{1}\left(\rho_{0}, \mu_{R}\right)\right|^{2} \Pi_{S_{+0}}\left(x_{0}, \rho_{0}, \rho_{1}\right) \\
& \times\left(1-\int_{\rho_{\mathrm{MS}}}^{\rho_{1}} d \rho d z \alpha_{\mathrm{S}}(\rho) \mathcal{P}_{2}(z, \rho) \Pi_{S_{+1}}\left(x_{1}, \rho_{1}, \rho\right)\right) \\
\frac{d \sigma_{2}^{i n}}{d \phi_{0}}= & d \rho_{1} d z_{1} d \rho_{2} d z_{2} f_{0}\left(x_{0}, \rho_{0}\right) \frac{\alpha_{\mathrm{S}}\left(\rho_{1}\right)}{\alpha_{\mathrm{s}}\left(\mu_{R}\right)} \frac{f_{1}\left(x_{1}, \rho_{1}\right)}{f_{0}\left(x_{0}, \rho_{1}\right)} \frac{\alpha_{\mathrm{S}}\left(\rho_{2}\right)}{\alpha_{\mathrm{S}}\left(\mu_{R}\right)} \frac{f_{2}\left(x_{2}, \rho_{2}\right)}{f_{1}\left(x_{1}, \rho_{2}\right)}\left|\mathcal{M}_{2}\left(\rho_{0}, \mu_{R}\right)\right|^{2} \\
& \Pi_{S_{+0}}\left(x_{0}, \rho_{0}, \rho_{1}\right) \Pi_{S_{+1}}\left(x_{1}, \rho_{1}, \rho_{2}\right)
\end{aligned}
$$

For a first-splitting-corrected PS all contributions not containing $\Pi_{S_{+1}}$ cancel between 3.7 and 3.9, except for the lowest order inclusive cross section. Unitarity is then guaranteed if

$$
\begin{gathered}
\int_{\rho_{1}}^{\rho_{0}} d \rho d z \int_{\rho_{\mathrm{MS}}}^{\rho_{1}} d \rho_{2} d z_{2} f_{0}\left(x_{0}, \rho_{0}\right) \frac{\alpha_{\mathrm{S}}\left(\rho_{1}\right)}{\alpha_{\mathrm{S}}\left(\mu_{R}\right)} \frac{f_{1}\left(x_{1}, \rho_{1}\right)}{f_{0}\left(x_{0}, \rho_{1}\right)}\left|\mathcal{M}_{1}\left(\rho_{0}, \mu_{R}\right)\right|^{2} \alpha_{\mathrm{S}}\left(\rho_{2}\right) \mathcal{P}_{2}\left(z_{2}, \rho_{2}\right) \\
\Pi_{S_{+0}}\left(x_{0}, \rho_{0}, \rho\right) \Pi_{S_{+1}}\left(x_{1}, \rho, \rho_{2}\right) \\
\int_{\rho_{1}}^{\rho_{0}} d \rho d z \int_{\rho_{\mathrm{MS}}}^{\rho_{1}} d \rho_{2} d z_{2} f_{0}\left(x_{0}, \rho_{0}\right) \frac{\alpha_{\mathrm{S}}(\rho)}{\alpha_{\mathrm{S}}\left(\mu_{R}\right)} \frac{f_{1}\left(x_{1}, \rho\right)}{f_{0}\left(x_{0}, \rho\right)} \frac{\alpha_{\mathrm{S}}\left(\rho_{2}\right)}{\alpha_{\mathrm{S}}\left(\mu_{R}\right)} \frac{f_{2}\left(x_{2}, \rho_{2}\right)}{f_{1}\left(x_{1}, \rho_{2}\right)}\left|\mathcal{M}_{2}\left(\rho_{0}, \mu_{R}\right)\right|^{2}(3 \\
\Pi_{S_{+0}}\left(x_{0}, \rho_{0}, \rho\right) \Pi_{S_{+1}}\left(x_{1}, \rho, \rho_{2}\right)
\end{gathered}
$$

For this, the splitting kernels need to exactly reproduce the matrix element, phase space must be fully covered by the parton shower, and the no-emission probabilities need to be produced identically in both cases. Particularly the requirement that the phase space is completely covered is problematic, since parton showers commonly fill only phase space regions in which consecutive emissions are ordered in a decreasing evolution variable.

Clearly, eq. (3.11) is not fulfilled in standard PS programs, which at best are correct to next-to-leading logarithmic (NLL) accuracy. This means that the dependence on the merging scale would vanish to order $\alpha_{\mathrm{s}}^{2} L^{4}$ and $\alpha_{\mathrm{S}}^{2} L^{3}$, but that there will be a residual logarithmic dependence of order $\alpha_{\mathrm{s}}^{2} L^{2}$.

In the next section, we would like to outline a method that sidesteps these problems by using multi-jet matrix elements from the very beginning to build the resummation for low-multiplicity states. 


\section{Concepts of UMEPS}

The main concept we would like to emphasise is that appropriately weighted matrix elements with additional jets can be used to induce resummation in lower-multiplicity states.

For example, one-jet inclusive cross sections (eq. (2.7)) can, by integrating over the phase space of the resolved jet, be manipulated to induce resummation in zero-jet cross section. No parton shower resummation above $\rho_{\mathrm{MS}}$ would then be necessary in zero-jet contributions. This means that we can reorder the parton shower formula for the inclusive cross section:

$$
\begin{aligned}
\sigma^{i n}= & \int d \phi_{0}\left(\frac{d \sigma_{0}^{e x}}{d \phi_{0}}+\int \frac{d \sigma_{1}^{i n}}{d \phi_{0}}\right) \\
= & \int d \phi_{0}\left(f_{0}\left(x_{0}, \rho_{0}\right)\left|\mathcal{M}_{0}\left(\rho_{0}, \mu_{R}\right)\right|^{2}\right. \\
& \quad \underbrace{f_{0}\left(x_{0}, \rho_{0}\right)\left|\mathcal{M}_{0}\left(\rho_{0}, \mu_{R}\right)\right|^{2} \int_{\rho_{\mathrm{MS}}}^{\rho_{0}} d \rho d z \alpha_{\mathrm{S}}(\rho) \mathcal{P}_{1}(z, \rho) \Pi_{S_{+0}}\left(x_{0}, \rho_{0}, \rho\right)}_{\frac{d \sigma_{1 \rightarrow 0}^{i n}}{d \phi_{0}}}+\int \frac{d \sigma_{1}^{i n}}{d \phi_{0}})
\end{aligned}
$$

and generate $\frac{d \sigma_{1 \rightarrow 0}^{i n}}{d \phi_{0}}$ explicitly from $\frac{d \sigma_{1}^{i n}}{d \phi_{0}}$ by integrating over the emission phase space. When including one additional jet into the parton shower, we can explicitly preserve the inclusive cross section by adding the samples

$$
\begin{aligned}
\frac{d \sigma_{0}^{i n}}{d \phi_{0}} & =f_{0}\left(x_{0}, \rho_{0}\right)\left|\mathcal{M}_{0}\left(\rho_{0}, \mu_{R}\right)\right|^{2} \\
\frac{d \sigma_{1}^{i n}}{d \phi_{0}} & =d \rho_{1} d z_{1} f_{0}\left(x_{0}, \rho_{0}\right) \frac{\alpha_{\mathrm{s}}\left(\rho_{1}\right)}{\alpha_{\mathrm{S}}\left(\mu_{R}\right)} \frac{f_{1}\left(x_{1}, \rho_{1}\right)}{f_{0}\left(x_{0}, \rho_{1}\right)}\left|\mathcal{M}_{1}\left(\rho_{0}, \mu_{R}\right)\right|^{2} \Pi_{S_{+0}}\left(x_{0}, \rho_{0}, \rho_{1}\right) \\
\frac{d \sigma_{1 \rightarrow 0}^{i n}}{d \phi_{0}} & =-\int_{\rho_{\mathrm{MS}}}^{\rho_{0}} d \rho d z f_{0}\left(x_{0}, \rho_{0}\right) \frac{\alpha_{\mathrm{S}}(\rho)}{\alpha_{\mathrm{s}}\left(\mu_{R}\right)} \frac{f_{1}\left(x_{1}, \rho\right)}{f_{0}\left(x_{0}, \rho\right)}\left|\mathcal{M}_{1}\left(\rho_{0}, \mu_{R}\right)\right|^{2} \Pi_{S_{+0}}\left(x_{0}, \rho_{0}, \rho\right)
\end{aligned}
$$

Before we continue, let us pause and investigate how we attach parton showers to these samples. In zero-jet contributions, the effect of parton showers above $\rho_{\mathrm{MS}}$ is already included, so that we only need to start the parton shower at $\rho_{\mathrm{MS}}$. If the one-jet matrix element is the highest multiplicity sample, we allow the shower to generate emissions below $\rho_{1}$, as in traditional merging. Since cross section changes from allowing e.g. two resolved jets cancel exactly with unresolved jets in the one-jet state (see eq. (2.10) and eq. (2.11) and the discussion following 2.12), allowing the shower to produce resolved emissions does not invalidate unitarity.

We call this method UMEPS, for unitary matrix element + parton shower merging. In principle, this method is as easily generalisable as traditional merging techniques, and shows, on a more detailed level, difficulties reminiscent of CKKW-L. To particularise, let us have a look at how two additional jets can be included by UMEPS. Naively, we would 
simply add

$$
\begin{aligned}
\frac{d \sigma_{2}^{i n}}{d \phi_{0}}=d \rho_{1} d z_{1} d \rho_{2} d z_{2} f_{0}\left(x_{0}, \rho_{0}\right) \frac{\alpha_{\mathrm{s}}\left(\rho_{1}\right)}{\alpha_{\mathrm{s}}\left(\mu_{R}\right)} \frac{f_{1}\left(x_{1}, \rho_{1}\right)}{f_{0}\left(x_{0}, \rho_{1}\right)} \frac{\alpha_{\mathrm{s}}\left(\rho_{2}\right)}{\alpha_{\mathrm{s}}\left(\mu_{R}\right)} \frac{f_{2}\left(x_{2}, \rho_{2}\right)}{f_{1}\left(x_{1}, \rho_{2}\right)}\left|\mathcal{M}_{2}\left(\rho_{0}, \mu_{R}\right)\right|^{2} \\
\quad \times \Pi_{S_{+0}}\left(x_{0}, \rho_{0}, \rho_{1}\right) \Pi_{S_{+1}}\left(x_{1}, \rho_{1}, \rho_{2}\right) \\
\frac{d \sigma_{2 \rightarrow 1}^{i n}}{d \phi_{0}}=-d \rho_{1} d z_{1} \int_{\rho_{\mathrm{MS}}}^{\rho_{1}} d \rho d z f_{0}\left(x_{0}, \rho_{0}\right) \frac{\alpha_{\mathrm{s}}\left(\rho_{1}\right)}{\alpha_{\mathrm{S}}\left(\mu_{R}\right)} \frac{f_{1}\left(x_{1}, \rho_{1}\right)}{f_{0}\left(x_{0}, \rho_{1}\right)} \frac{\alpha_{\mathrm{s}}\left(\rho_{2}\right)}{\alpha_{\mathrm{S}}\left(\mu_{R}\right)} \frac{f_{2}\left(x_{2}, \rho\right)}{f_{1}\left(x_{1}, \rho\right)}\left|\mathcal{M}_{2}\left(\rho_{0}, \mu_{R}\right)\right|^{2} \\
\times \Pi_{S_{+0}}\left(x_{0}, \rho_{0}, \rho_{1}\right) \Pi_{S_{+1}}\left(x_{1}, \rho_{1}, \rho\right)
\end{aligned}
$$

and treat 4.5 as highest multiplicity sample. It is however possible that due to undoing recoil effects, states with jets below $\rho_{\mathrm{MS}}$ are produced by performing the integration in 4.6. In this case, we take these contributions to be corrections to the zero-jet cross section, and integrate twice. After this amendment, two-jet UMEPS merging contains the contributions

$$
\begin{aligned}
& \frac{d \sigma_{0}^{i n}}{d \phi_{0}}=f_{0}\left(x_{0}, \rho_{0}\right)\left|\mathcal{M}_{0}\left(\rho_{0}, \mu_{R}\right)\right|^{2} \\
& \frac{d \sigma_{1}^{i n}}{d \phi_{0}}=d \rho_{1} d z_{1} f_{0}\left(x_{0}, \rho_{0}\right) \frac{\alpha_{\mathrm{s}}\left(\rho_{1}\right)}{\alpha_{\mathrm{s}}\left(\mu_{R}\right)} \frac{f_{1}\left(x_{1}, \rho_{1}\right)}{f_{0}\left(x_{0}, \rho_{1}\right)}\left|\mathcal{M}_{1}\left(\rho_{0}, \mu_{R}\right)\right|^{2} \Pi_{S_{+0}}\left(x_{0}, \rho_{0}, \rho_{1}\right) \\
& \frac{d \sigma_{1 \rightarrow 0}^{i n}}{d \phi_{0}}=-\int_{\rho_{\mathrm{MS}}}^{\rho_{0}} d \rho d z f_{0}\left(x_{0}, \rho_{0}\right) \frac{\alpha_{\mathrm{s}}(\rho)}{\alpha_{\mathrm{S}}\left(\mu_{R}\right)} \frac{f_{1}\left(x_{1}, \rho\right)}{f_{0}\left(x_{0}, \rho\right)}\left|\mathcal{M}_{1}\left(\rho_{0}, \mu_{R}\right)\right|^{2} \Pi_{S_{+0}}\left(x_{0}, \rho_{0}, \rho\right) \\
& \frac{d \sigma_{2}^{i n}}{d \phi_{0}}=d \rho_{1} d z_{1} d \rho_{2} d z_{2} f_{0}\left(x_{0}, \rho_{0}\right) \frac{\alpha_{\mathrm{S}}\left(\rho_{1}\right)}{\alpha_{\mathrm{s}}\left(\mu_{R}\right)} \frac{f_{1}\left(x_{1}, \rho_{1}\right)}{f_{0}\left(x_{0}, \rho_{1}\right)} \frac{\alpha_{\mathrm{s}}\left(\rho_{2}\right)}{\alpha_{\mathrm{S}}\left(\mu_{R}\right)} \frac{f_{2}\left(x_{2}, \rho_{2}\right)}{f_{1}\left(x_{1}, \rho_{2}\right)}\left|\mathcal{M}_{2}\left(\rho_{0}, \mu_{R}\right)\right|^{2} \\
& \Pi_{S_{+0}}\left(x_{0}, \rho_{0}, \rho_{1}\right) \Pi_{S_{+1}}\left(x_{1}, \rho_{1}, \rho_{2}\right) \\
& \frac{d \sigma_{2 \rightarrow 1}^{i n}}{d \phi_{0}}=-d \rho_{1} d z_{1} \int_{\rho_{\mathrm{MS}}}^{\rho_{1}} d \rho d z f_{0}\left(x_{0}, \rho_{0}\right) \frac{\alpha_{\mathrm{S}}\left(\rho_{1}\right)}{\alpha_{\mathrm{S}}\left(\mu_{R}\right)} \frac{f_{1}\left(x_{1}, \rho_{1}\right)}{f_{0}\left(x_{0}, \rho_{1}\right)} \frac{\alpha_{\mathrm{S}}(\rho)}{\alpha_{\mathrm{S}}\left(\mu_{R}\right)} \frac{f_{2}\left(x_{2}, \rho\right)}{f_{1}\left(x_{1}, \rho\right)}\left|\mathcal{M}_{2}\left(\rho_{0}, \mu_{R}\right)\right|^{2} \\
& \Theta\left(\rho_{1}-\rho_{\mathrm{MS}}\right) \Pi_{S_{+0}}\left(x_{0}, \rho_{0}, \rho_{1}\right) \Pi_{S_{+1}}\left(x_{1}, \rho_{1}, \rho\right) \\
& \frac{d \sigma_{2 \rightarrow 0}^{i n}}{d \phi_{0}}=-\int_{\rho_{1}}^{\rho_{0}} d \rho_{a} d z_{a} \int_{\rho_{\mathrm{MS}}}^{\rho_{1}} d \rho_{b} d z_{b} f_{0}\left(x_{0}, \rho_{0}\right) \frac{\alpha_{\mathrm{S}}\left(\rho_{a}\right)}{\alpha_{\mathrm{S}}\left(\mu_{R}\right)} \frac{f_{1}\left(x_{1}, \rho_{a}\right)}{f_{0}\left(x_{0}, \rho_{a}\right)} \frac{\alpha_{\mathrm{S}}\left(\rho_{b}\right)}{\alpha_{\mathrm{S}}\left(\mu_{R}\right)} \frac{f_{2}\left(x_{2}, \rho_{b}\right)}{f_{1}\left(x_{1}, \rho_{b}\right)}\left|\mathcal{M}_{2}\left(\rho_{0}, \mu_{R}\right)\right|^{2} \\
& \Theta\left(\rho_{\mathrm{MS}}-\rho_{a}\right) \Pi_{S_{+0}}\left(x_{0}, \rho_{0}, \rho_{a}\right) \Pi_{S_{+1}}\left(x_{1}, \rho_{a}, \rho_{b}\right)
\end{aligned}
$$

UMEPS can then be extended to arbitrary jet multiplicity. The main idea is that in order keep unitarity, we have to subtract all contributions that we add as higher multiplicity matrix elements. The subtractions are constructed with PS unitarity as a guideline. For brevity, we introduce the short-hands

$$
\frac{d \sigma_{n}^{i n}}{d \phi_{0}}=\mathrm{B}_{n} w_{n}^{\prime}=\widehat{\mathrm{B}}_{n} \quad \text { and } \quad \frac{d \sigma_{n \rightarrow m}^{i n}}{d \phi_{0}}=-\int d^{n-m} \phi \mathrm{B}_{n} w_{n}^{\prime}=-\int_{s} \widehat{\mathrm{B}}_{n \rightarrow m}
$$

where $w_{n}^{\prime}$ will be defined below. The symbol $\int_{s} \widehat{\mathrm{B}}_{n \rightarrow m}$ indicates that more than one integrations had to be performed since all of the states $S_{+n-1}, \ldots, S_{+m+1}$ contained partons below the merging scale. The integration(s) will be achieved by substituting the input event with a reconstructed lower-multiplicity event of the parton shower history, as will be discussed in section 4.1. This substitution method is indicated by the subscript $s$ on the integral sign. The weight $w_{n}^{\prime}$ that needs to be applied to tree-level events to produce the 
$\frac{d \sigma_{n}^{i n}}{d \phi}$ sample is given by

$$
\begin{aligned}
w_{n}^{\prime}= & \frac{x_{n} f_{n}\left(x_{n}, \rho_{n}\right)}{x_{n} f_{n}\left(x_{n}, \mu_{F}\right)} \times \prod_{i=1}^{n}\left[\frac{\alpha_{\mathrm{s}}\left(\rho_{i}\right)}{\alpha_{\mathrm{S}}\left(\mu_{R}\right)} \frac{x_{i-1} f_{i-1}\left(x_{i-1}, \rho_{i-1}\right)}{x_{i-1} f_{i-1}\left(x_{i-1}, \rho_{i}\right)} \Pi_{S_{+i-1}}\left(x_{i-1}, \rho_{i-1}, \rho_{i}\right)\right] \\
= & \frac{x_{n}^{+} f_{n}^{+}\left(x_{n}^{+}, \rho_{n}\right)}{x_{n}^{+} f_{n}^{+}\left(x_{n}^{+}, \mu_{F}\right)} \frac{x_{n}^{-} f_{n}^{-}\left(x_{n}^{-}, \rho_{n}\right)}{x_{n}^{-} f_{n}^{-}\left(x_{n}^{-}, \mu_{F}\right)} \\
& \quad \times \prod_{i=1}^{n}\left[\frac{\alpha_{\mathrm{s}}\left(\rho_{i}\right)}{\alpha_{\mathrm{s}}\left(\mu_{R}\right)} \frac{x_{i-1}^{+} f_{i-1}^{+}\left(x_{i-1}^{+}, \rho_{i-1}\right)}{x_{i-1}^{+} f_{i-1}^{+}\left(x_{i-1}^{+}, \rho_{i}\right)} \frac{x_{i-1}^{-} f_{i-1}^{-}\left(x_{i-1}^{-}, \rho_{i-1}\right)}{x_{i-1}^{-} f_{i-1}^{-}\left(x_{i-1}^{-}, \rho_{i}\right)} \Pi_{S_{+i-1}}\left(x_{i-1}, \rho_{i-1}, \rho_{i}\right)\right] .
\end{aligned}
$$

This weight differs from the CKKW-L weight in eq. (3.2), since it does not contain the last no-emission probability $\Pi_{S_{+n}}\left(x_{n}, \rho_{n}, \rho_{\mathrm{MS}}\right)$, i.e. the last line in eq. (3.2). In the UMEPS procedure, this factor is instead included by subtracting the integrated, reweighted, nexthigher multiplicity sample, thus conserving unitarity in a way reminiscent of standard parton showers. The probability of having no resolved emissions off the zero-jet states in eq. (4.7) for example, is included through the contributions in eqs. (4.9) and eq. (4.12).

Armed with this notation, the prediction of an observable $\mathcal{O}$ in 2 -jet merged UMEPS becomes

$$
\begin{aligned}
\langle\mathcal{O}\rangle=\int d \phi_{0} & \left\{\mathcal{O}\left(S_{+0 j}\right) \widehat{\mathrm{B}}_{0}-\mathcal{O}\left(S_{+0 j}\right) \int_{s} \widehat{\mathrm{B}}_{1 \rightarrow 0}-\mathcal{O}\left(S_{+0 j}\right) \int_{s} \widehat{\mathrm{B}}_{2 \rightarrow 0}\right. \\
& +\int \mathcal{O}\left(S_{+1 j}\right) \widehat{\mathrm{B}}_{1}-\int \mathcal{O}\left(S_{+1 j}\right) \int_{s} \widehat{\mathrm{B}}_{2 \rightarrow 1} \\
& \left.+\iint \mathcal{O}\left(S_{+2 j}\right) \widehat{\mathrm{B}}_{2}\right\}
\end{aligned}
$$

where we have used the notation $S_{+n j}$ to indicate states with $n$ resolved jets, resolved meaning above the cut $\rho_{\mathrm{MS}}$ as defined by the merging scale definition. More generally, the outcome of merging $n$ additional partons with the UMEPS method is

$$
\begin{aligned}
&\langle\mathcal{O}\rangle=\int d \phi_{0}\left\{\mathcal{O}\left(S_{+0 j}\right)\left[\widehat{\mathrm{B}}_{0}-\int_{s} \widehat{\mathrm{B}}_{1 \rightarrow 0}-\int_{s} \widehat{\mathrm{B}}_{2 \rightarrow 0}-\ldots-\int_{s} \widehat{\mathrm{B}}_{N \rightarrow 0}\right]\right. \\
&+\int \mathcal{O}\left(S_{+1 j}\right)\left[\widehat{\mathrm{B}}_{1}-\int_{s} \widehat{\mathrm{B}}_{2 \rightarrow 1}-\ldots-\int_{s} \widehat{\mathrm{B}}_{N \rightarrow 1}\right] \\
&+\ldots \\
&+\int \ldots \int \mathcal{O}\left(S_{+N-1 j}\right)\left[\widehat{\mathrm{B}}_{N-1}-\int_{s} \widehat{\mathrm{B}}_{N \rightarrow N-1}\right] \\
&\left.+\int \ldots \int \mathcal{O}\left(S_{+N j}\right) \widehat{\mathrm{B}}_{N}\right\} \\
&=\sum_{n=0}^{N} \int d \phi_{0} \int \ldots \int \mathcal{O}\left(S_{+n j}\right)\left\{\widehat{\mathrm{B}}_{n}-\sum_{i=n+1}^{N} \int_{s} \widehat{\mathrm{B}}_{i \rightarrow n}\right\} .
\end{aligned}
$$

The generation of $\widehat{\mathrm{B}}_{n}-$ and $\int_{s} \widehat{\mathrm{B}}_{n \rightarrow m}$ - events will be summarised in section 4.1. It should be noted that the treatment $\rho_{\mathrm{MS}}$-unordered integration results is heavily influenced by how 
CKKW-L includes states with $\rho_{\mathrm{MS}}$-unordered emissions, which was discussed in detail in [16]. Precisely for states which evolve from a state below $\rho_{\mathrm{MS}}$ to a state above $\rho_{\mathrm{MS}}$ do CKKW-L and the truncated-shower [24] approach differ. It can thus be imagined that other ways of treating such notorious configurations show improved behaviour. For now, we will not discuss such possibilities, and instead, if necessary, integrate multiple times, until a state above $\rho_{\mathrm{MS}}$ is produced.

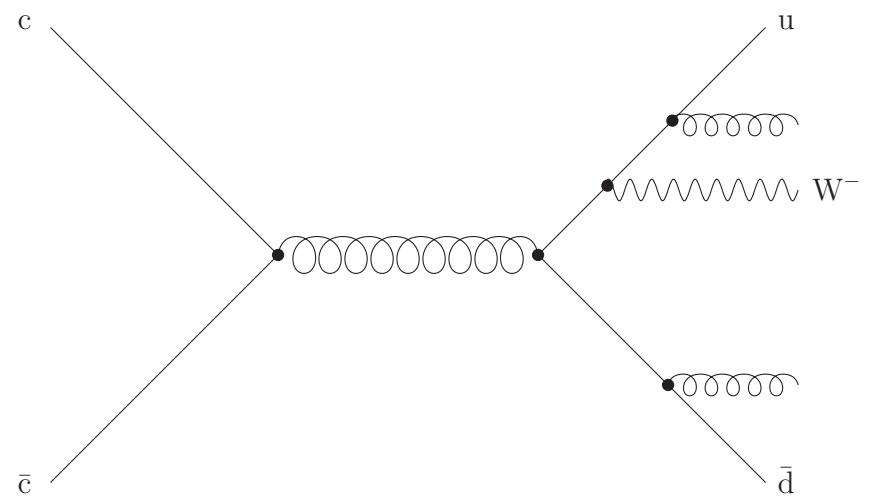

Figure 1: An example of a matrix element contribution without a complete shower history. If the parton shower does not include $\mathrm{W}$-boson radiation, only the two gluon emissions can be reclustered, and $c \overline{\mathrm{c}} \rightarrow \mathrm{u} \overline{\mathrm{d}} \mathrm{W}^{-}$has to be considered a separate hard process.

A well-known challenge of merging prescriptions is the treatment of configurations that could never have been produced by a sequence of shower splittings. This can happen if the PS does not include all possible splittings of the model. Figure f for example cannot be produced by a shower that does not allow W-boson radiation. Such states cannot be projected onto a lower-multiplicity underlying process, and will thus introduce small unitarity violations in UMEPS.

\subsection{Procedure step-by-step}

To implement UMEPS, we need to be able to perform the necessary integrations. Although the formulae could convey a feeling of complexity, these integrations are factually already needed in traditional merging approaches. All modern CKKW-inspired schemes need to construct a history of parton shower states for input matrix element events, because otherwise, no trial showers can be used to generate Sudakov form factors dynamically. Thus, a sequence of states $S_{+n} \rightarrow S_{+n-1} \rightarrow \cdots \rightarrow S_{+1} \rightarrow S_{+0}$ is always available. The sequence is constructed by inverting the shower mapping of radiative phase space on each state, i.e. a parton in $S_{+n}$ is removed, and its momentum distributed amongst the remaining particles, leading to a state $S_{+n-1}$. This is exactly the integration we need: To produce the integrated version of $S_{+n}$, we simply replace it by $S_{+n-1}$, but keep the full weight. $k$-fold integrations can be achieved by replacing $S_{+n}$ with $S_{+n-k}$.

With this, we have all ingredients to construct the UMEPS scheme. If not mentioned explicitly, all weights in UMEPS are generated precisely as in CKKW-L. To avoid unnec-

\footnotetext{
${ }^{4}$ The mapping used for the current paper is given in Appendix B.2 of [16]
} 
essary complications, we will here gloss over many technicalities that have already been addressed in CKKW-L, and are directly borrowed by UMEPS. A discussion of Sudakov reweighting for states without parton-shower ordered histories, for example, can be found in $[16]$.

The UMEPS algorithm has two parts - a part in which we keep the matrix element configurations $\left(\widehat{\mathrm{B}}_{n}-\right.$ events) and another in which we integrate over emissions $\left(\int_{s} \widehat{\mathrm{B}}_{n \rightarrow m}\right.$ - events):

I. Produce Les Houches event files (LHEF) [25] with a matrix element generator for $n=0,1 \ldots N$ extra jets with a regularisation cut-off, $\rho_{\mathrm{MS}}$, typically using a fixed factorisation scale, $\mu_{F}$, and a fixed $\alpha_{\mathrm{s}}\left(\mu_{R}\right)$.

II. Pick a jet multiplicity, $n$, and a state $S_{+n}$ according to the cross sections given by the matrix element generator.

1. Find all shower histories for the state $S_{+n}$, pick a sequence according to the product of splitting probabilities. Only pick un-ordered sequences if no ordered sequence was found. Only pick incomplete paths if no complete path was constructed.

2. Perform reweighting: For each $0 \leqslant i<n$,

i. Start the shower off the state $S_{+i}$ at $\rho_{i}$, generate a trial state $R$ with scale $\rho_{R}$. If $\rho_{R}>\rho_{i+1}$, veto the event and start again from II.

ii. Calculate the weight factor

$$
w_{i}=\frac{\alpha_{\mathrm{s}}\left(\rho_{i+1}\right)}{\alpha_{\mathrm{s}}\left(\mu_{R}\right)} \frac{x_{i}^{+} f_{i}^{+}\left(x_{i}^{+}, \rho_{i}\right)}{x_{i}^{+} f_{i}^{+}\left(x_{i}^{+}, \rho_{i+1}\right)} \frac{x_{i}^{-} f_{i}^{-}\left(x_{i}^{-}, \rho_{i}\right)}{x_{i}^{-} f_{i}^{-}\left(x_{i}^{-}, \rho_{i+1}\right)}
$$

3. Start the shower from $S_{+n}$.

i. If $n<N$, start the shower at $\rho_{n}$, veto any shower emission producing an additional resolved jet.

ii. If $n=N$, start the shower at $\rho_{n}$.

III. If the event was not rejected, multiply the event weight by

$$
w_{n}^{\prime}=\frac{x_{n}^{+} f_{n}^{+}\left(x_{n}^{+}, \rho_{n}\right)}{x_{n}^{+} f_{n}^{+}\left(x_{n}^{+}, \mu_{F}\right)} \times \frac{x_{n}^{-} f_{n}^{-}\left(x_{n}^{-}, \rho_{n}\right)}{x_{n}^{-} f_{n}^{-}\left(x_{n}^{-}, \mu_{F}\right)} \times \prod_{i=0}^{n-1} w_{i}
$$

\section{Start again from II.}

The second part, i.e. producing $\int_{s} \widehat{\mathrm{B}}_{n \rightarrow m}$ - events to effect lower-multiplicity PS resummation, requires only two changes:

II.3 Replace the matrix-element state by $S_{+n-1}$, or the first state $S_{+l}$ with all $l \leq n-1$ partons above the merging scale. If no integrated state can be constructed, i.e. if only incomplete paths were found, reject the event. For valid events, start the shower at $\rho_{n}$, veto any shower emission producing an additional resolved jet. 
III. If the event was not rejected, multiply the event weight by

$$
-w_{n}^{\prime}=-\frac{x_{n}^{+} f_{n}^{+}\left(x_{n}^{+}, \rho_{n}\right)}{x_{n}^{+} f_{n}^{+}\left(x_{n}^{+}, \mu_{F}\right)} \times \frac{x_{n}^{-} f_{n}^{-}\left(x_{n}^{-}, \rho_{n}\right)}{x_{n}^{-} f_{n}^{-}\left(x_{n}^{-}, \mu_{F}\right)} \times \prod_{i=0}^{n-1} w_{i}
$$

Finally, all samples generated in the first and second parts are added to give the UMEPS prediction. Note that in order produce correctly normalised cross sections $\frac{d \sigma_{i}^{e x}}{d \phi_{0}}$, we need to include ratios of parton distributions and $\alpha_{\mathrm{s}}$ ratios into the weight. This is analogous to the CKKW-L method (see the $\alpha_{\mathrm{s}^{-}}$and PDF-factors in eq. (3.2)). It is worthwhile to notice that the UMEPS scheme can in principle be implemented by using structures already existing in traditional merging codes. Basically, compared to traditional merging, the $\widehat{\mathrm{B}}_{n}$-contributions do not carry a no-emission probability for emissions off the ME event. The $\int_{s} \widehat{\mathrm{B}}_{n \rightarrow m}$-samples can easily be extracted from merging codes.

How multiple partonic interactions (MPI) are added to the merged samples requires a short discussion. In principle, we stay true to the philosophy of the algorithm outlined in [16], i.e. we want to make sure that the merging method does not artificially suppress hard secondary scatterings, which in PYTHIA8 are interleaved with the parton shower. The interleaving means that the PS is competing with the MPI's, and the probability of emitting a parton in the PS is not only governed by the standard no-emission probability, $\Pi_{S_{+n}}$ but is also multiplied by a no-MPI probability, $\Pi_{S_{+n}}^{\mathrm{MPI}}$. Hence all $m$-jet (both $\widehat{\mathrm{B}}_{m}$ and $\int_{s} \widehat{\mathrm{B}}_{n \rightarrow m}$ ) samples generated by our algorithm above need to be multiplied by the no-MPI probabilities

$$
\prod_{i=0}^{m-1} \Pi_{S_{+i}}^{\mathrm{MPI}}\left(\rho_{i}, \rho_{i+1}\right)
$$

which are easily incorporated in the trial showers described above. We also need to include the actual MPI's. Here the philosophy is that as soon as we have a MPI at some scale, we ignore corrections from the full tree-level matrix element on softer jets from the primary interaction, and allow them to be described by the PS alone. Hence, when we start the shower from a given $m$-parton state (with $m<N$ ) in step II.3, we choose the reconstructed $\rho_{m}$ as starting scale. As described before, we veto any parton emission above $\rho_{\mathrm{MS}}$. However, if a MPI is generated above $\rho_{\text {MS }}$, it is accepted and the shower is allowed to continue without any further veto. (For the $m=N$ case, the shower including MPI is allowed without restrictions, starting from $\rho_{N}$.) In this way we achieve the same goal as in [16]: If the $n \leq N$ hardest jets in an event all belong to the primary interaction, they are described by the tree-level ME, while all other jets are given by the (interleaved) PS. Just as in [16], the treatment of pure QCD jet production means that the Born-level cross section is properly eikonalized by the no-MPI factor, by allowing MPI's all the way from $\sqrt{s}$ in the trial shower for $\Pi_{S_{+0}}^{\mathrm{MPI}}$.

\section{Results}

We have implemented UMEPS merging in PYTHIA8, and will make the necessary code public in the next major release version. In this section, we will concentrate on predictions 
for W-boson and QCD jet production at the LHC. However, the code aims to achieve the same generality as the implementation of CKKW-L in PYTHIA8.

All input matrix element configurations are taken from Les Houches Event Files generated with MadGraph/MadEvent, with the following settings:

- Fixed renormalisation scale $\mu_{R}=\mathrm{M}_{\mathrm{Z}}^{2}$, fixed factorisation scale $\mu_{F}=\mathrm{M}_{\mathrm{W}}^{2}$ for $\mathrm{W}$ production. For $2 \rightarrow 2$ processes in pure QCD, we use $\mu_{r, 2 \rightarrow 2}=m_{\perp, 1} m_{\perp, 1}$ and $\mu_{f, 2 \rightarrow 2}=\min \left\{m_{\perp, 1}^{2}, m_{\perp, 1}^{2}\right\}$.

- CTEQ6L1 parton distributions and $\alpha_{\mathrm{S}}\left(\mathrm{M}_{\mathrm{Z}}^{2}\right)=0.130$.

- The merging scale $\rho_{\mathrm{MS}}$ is defined by the minimal PYTHIA8 evolution $p_{\perp, i j k}$ of all possible combinations of three partons in the event. $p_{\perp, i j k}$ for a single combination of three particles $i, j$ and $k$ is defined as

$$
p_{\perp, i j k}^{2}= \begin{cases}z_{i j k}\left(1-z_{i j k}\right) Q_{i j}^{2} & \text { with } Q_{i j}^{2}=\left(p_{i}+p_{j}\right)^{2}, z_{i j k}=\frac{x_{i, j k}}{x_{i, j k}+x_{j, i k}}, \\ & x_{i, j k}=\frac{2 p_{i}\left(p_{i}+p_{j}+p_{k}\right)}{\left(p_{i}+p_{j}+p_{k}\right)^{2}} \quad \text { for FSR } \\ \left(1-z_{i j k}\right) Q_{i j}^{2} \quad & \text { with } Q_{i j}^{2}=-\left(p_{i}-p_{j}\right)^{2}, z_{i j k}=\frac{\left(p_{i}-p_{j}+p_{k}\right)^{2}}{\left(p_{i}+p_{k}\right)^{2}} \\ & \text { for ISR }\end{cases}
$$

- In QCD $2 \rightarrow 2$ scatterings, the kinematical transverse momentum of jets is required to be larger than $p_{T, j}=5 \mathrm{GeV}$.

The value of $\alpha_{\mathrm{s}}\left(\mathrm{M}_{\mathrm{Z}}^{2}\right)$ was set to match the $\alpha_{\mathrm{s}}$-value obtained in fitting the PDFs used in the ME calculation. To generate results, we have chosen the merging scale definition to closely match the parton shower evolution variable. The algorithm does however not depend on this particular choice. All jets needed for analysis purposes were defined with help of fastjet-routines [26]. The momentum of the intermediate W-boson will, if required, be extracted directly from the Monte Carlo event. We will compare UMEPS to the CKKW-L implementation in PYTHIA8. The problems we choose to highlight should be regarded as criticism of the implementation in PYTHIA8, rather than an assessment of CKKW-inspired methods in general.

\subsection{W-boson production}

We begin by comparing the result of the removal of a jet by integration with the corresponding parton shower contribution. This is useful to assess if performing the integration by the replacement $S_{+n+1} \rightarrow S_{+n}$ produces the desired results.

In the left panel of Figure 2, we compare the integrated one-jet matrix element (i.e. the $\mathcal{O}\left(\alpha_{\mathrm{s}}^{1}\left(\mu_{R}\right)\right)$-term of eq. (4.9)) with the shower approximation of the $\mathcal{O}\left(\alpha_{\mathrm{s}}^{1}\left(\mu_{R}\right)\right)$-term in zero-jet events. The second term is of course just the $\mathcal{O}\left(\alpha_{\mathrm{s}}^{1}\left(\mu_{R}\right)\right)$-contribution in eq. (3.6). The rapidity of the $\mathrm{W}$-boson is identical in these two samples because PYTHIA 8 is already matrix-element corrected for $\mathrm{W}+j$-states. This demonstrates that in $\mathrm{W}$-boson production, generating the no-emission probability in zero-jet states with PYTHIA8, or by a reweighted, integrated one-jet matrix element are both legitimate ways to produce the same factor. 

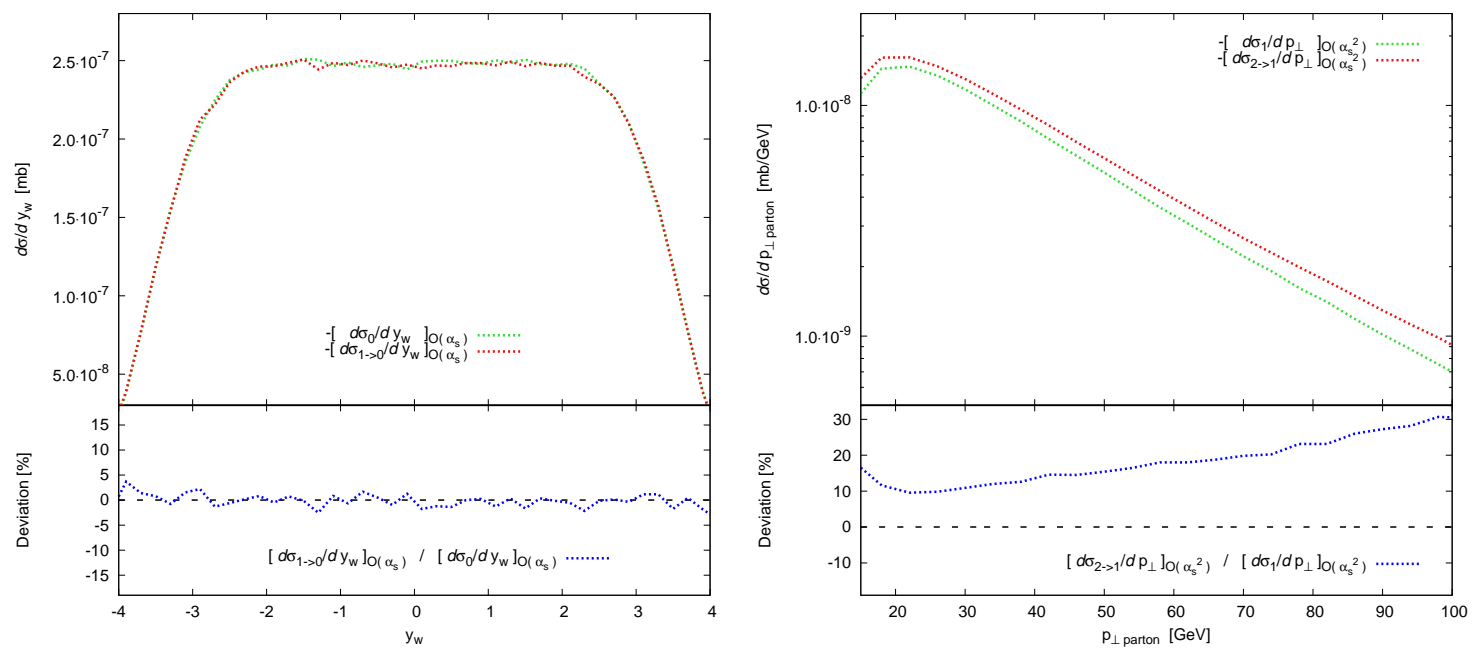

Figure 2: Comparison between $\mathcal{O}\left(\alpha_{\mathrm{s}}\right)$-terms of the parton shower with integrated matrix elements, for W-boson production in pp collisions at $E_{\mathrm{CM}}=7000 \mathrm{GeV}$. The merging scale value is $\rho_{\mathrm{MS}}=15 \mathrm{GeV}$. Left panel: Rapidity of the W-boson, intended for comparison between the integrated one-jet matrix element (labelled $\left.\left[d \sigma_{1 \rightarrow 0} / d y_{w}\right]_{\mathcal{O}\left(\alpha_{\mathrm{s}}^{1}\right)}\right)$ and the $\mathcal{O}\left(\alpha_{\mathrm{s}}^{1}\right)$-term of the noemission probability for having no emission above $\rho_{\text {MS }}$ radiated off zero-jet states above $\rho_{\text {MS }}$ (labelled $\left.\left[d \sigma_{0} / d y_{w}\right]_{\mathcal{O}\left(\alpha_{s}^{1}\right)}\right)$. Right panel: Transverse momentum of the parton, for $\mathrm{W}+j$ production in pp collisions at $E_{\mathrm{CM}}=7000 \mathrm{GeV}$, intended for comparison between the integrated two-jet matrix element (labelled $\left.\left[d \sigma_{2 \rightarrow 1} / d p_{\perp}\right]_{\mathcal{O}\left(\alpha_{\mathrm{s}}^{2}\right)}\right)$ and the $\mathcal{O}\left(\alpha_{\mathrm{s}}^{1}\right)$-term of the no-emission probability for having no emission above $\rho_{\mathrm{MS}}$ radiated off one-jet states, multiplying the $\mathrm{W}+j$ matrix element (labelled $\left.\left[d \sigma_{1} / d p_{\perp}\right]_{\mathcal{O}\left(\alpha_{\mathrm{s}}^{2}\right)}\right)$.

The right panel of Figure 2 investigates the difference between the parton shower approximation of no resolved emissions in one-jet states between the scales $\rho_{1}$ and $\rho_{\mathrm{MS}}$ and the result of constructing an unresolved emission by integrating over one parton in a twojet matrix element. This means that we compare the one-jet matrix element, multiplied by the $\mathcal{O}\left(\alpha_{\mathrm{s}}^{1}\left(\mu_{R}\right)\right)$-term of the no-emission probability $\Pi_{S_{+1}}\left(x_{1}, \rho_{1}, \rho_{\mathrm{MS}}\right)$ in eq. (3.8), with the $\mathcal{O}\left(\alpha_{\mathrm{s}}^{2}\left(\mu_{R}\right)\right)$-contribution in eq. (4.11). The comparison shows that, as expected, the parton shower underestimates the hardness of the unresolved (second) emission, which is reminiscent of the fact that the inclusion of two-jet matrix elements into the PS prediction does in general increase the tail of the $p_{\perp}$ of the hardest jet.

In Figure, 3 we show how matrix element samples contribute to this increase. All jet multiplicities enter, because the merging scale is not defined as the jet-separation of the $k_{\perp}$-algorithm, and since the merging scale cut acts on the matrix element state, while the jets are constructed from outgoing particles after the parton shower cascade. In CKKW-L, the high- $p_{\perp}$ tail is dominated by the two-jet matrix element, with a major contribution from the one-jet states. The latter is significantly lower in UMEPS, a fact that we think crucial. UMEPS correctly cancels the inclusion of phase space points with two resolved jets 

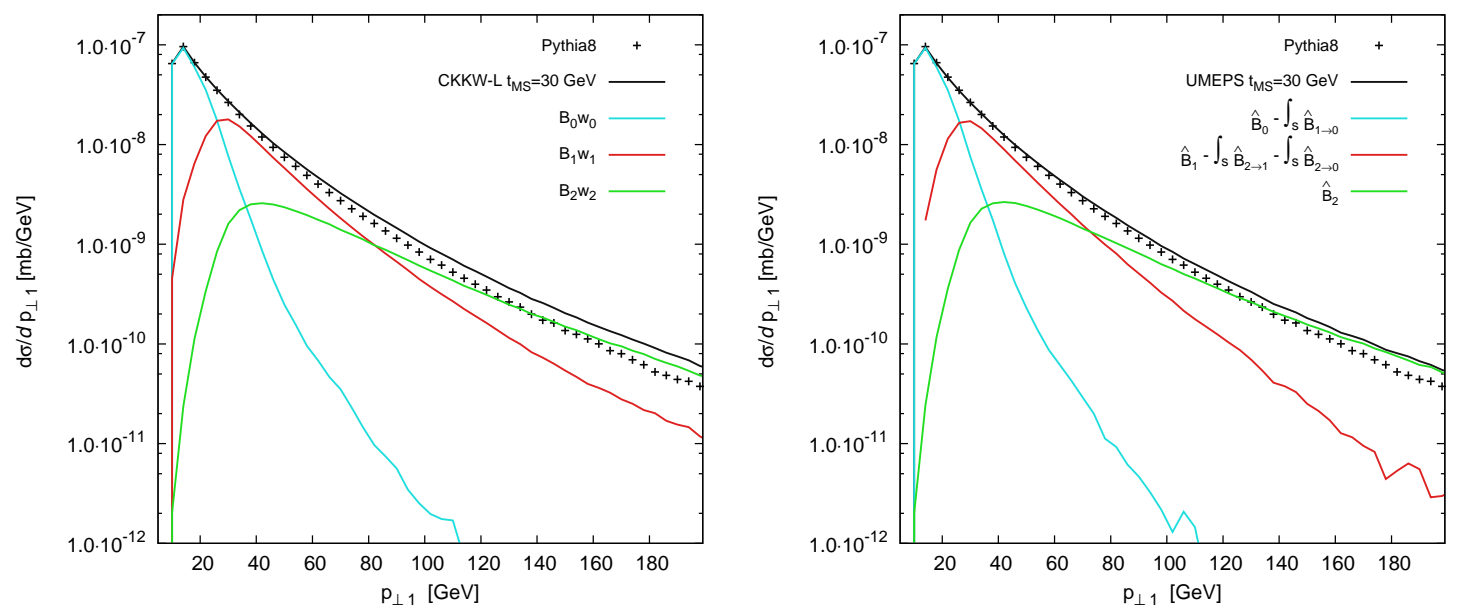

Figure 3: Transverse momentum of the hardest jet, for W-boson production in pp collisions at $E_{\mathrm{CM}}=7000 \mathrm{GeV}$, when merging up to two additional partons. Jets were defined with the $k_{\perp}$ algorithm, with $k_{\perp, \text { min }}=10 \mathrm{GeV}$. Multi-parton interactions and hadronisation were excluded. Left panel: Results of the CKKW-L scheme. The contributions are labelled $\mathrm{B}_{0} w_{0}, \mathrm{~B}_{1} w_{1}$ and $\mathrm{B}_{2} w_{2}$ for CKKW-L-reweighted zero-, one- and two-jet matrix elements, respectively. Right panel: Results of the UMEPS scheme. The contributions are labelled $\widehat{\mathrm{B}}_{0}, \widehat{\mathrm{B}}_{1}$ and $\widehat{\mathrm{B}}_{2}$ for UMEPS-reweighted zero-, one- and two-jet matrix elements, and $\int_{s} \widehat{B}_{1 \rightarrow 0}$ and $\int_{s} \widehat{B}_{2 \rightarrow 1}$ for UMEPS-reweighted, integrated oneand two-jet samples. $\int_{s} \widehat{\mathrm{B}}_{2 \rightarrow 0}$ indicates the two-jet contribution that was integrated twice because the state $S_{+1}$ after the first integration contained an unresolved jet.

by using the two-jet matrix element to construct a better approximation of radiating an unresolved parton from one-jet states. We see both in CKKW-L (left panel of Figure 3), and in Figure 2, that the parton shower underestimates the hardness of two-parton states. The description of unresolved emissions enters into the no-emission probabilities, with a negative $\mathcal{O}\left(\alpha_{\mathrm{s}}^{1}\right)$-term. Thus, the contribution of showered one-jet states to the tail of $p_{\perp 1}$ will be larger if the shower description of two-jet states underestimates hardness. UMEPS improves the description of the no-emission probability by ensuring that in inclusive observables, resolved two-jet states are cancelled, a feature that is at work in the tail of $p_{\perp 1}$.

Variations in the description of $p_{\perp 1}$ are also visible in Figure 4 , where we show the transverse momentum of the hardest jet in CKKW-L and UMEPS ${ }^{5}$. The trend sketched in the previous paragraph is particularly clear in the insets comparing to default PYTHIA8: UMEPS produces a softer tail in $p_{\perp 1}$ than CKKW-L. The harder tail in CKKW-L is due to a worse description of unresolved emissions. It is fair to say that the difference between CKKW-L and UMEPS hints at the size of relic effects from not cancelling the higher-multiplicity matrix elements in a well-defined way. Merging scale variations in treelevel merging schemes arise from a mismatch of unresolved emissions exponentiated in no-emission probabilities and tree-level matrix elements for hard, resolved jets. UMEPS

\footnotetext{
${ }^{5}$ Note that the co-variation of merged results in the ratio inset is due to fluctuations in the PYTHIA8 reference curve.
} 

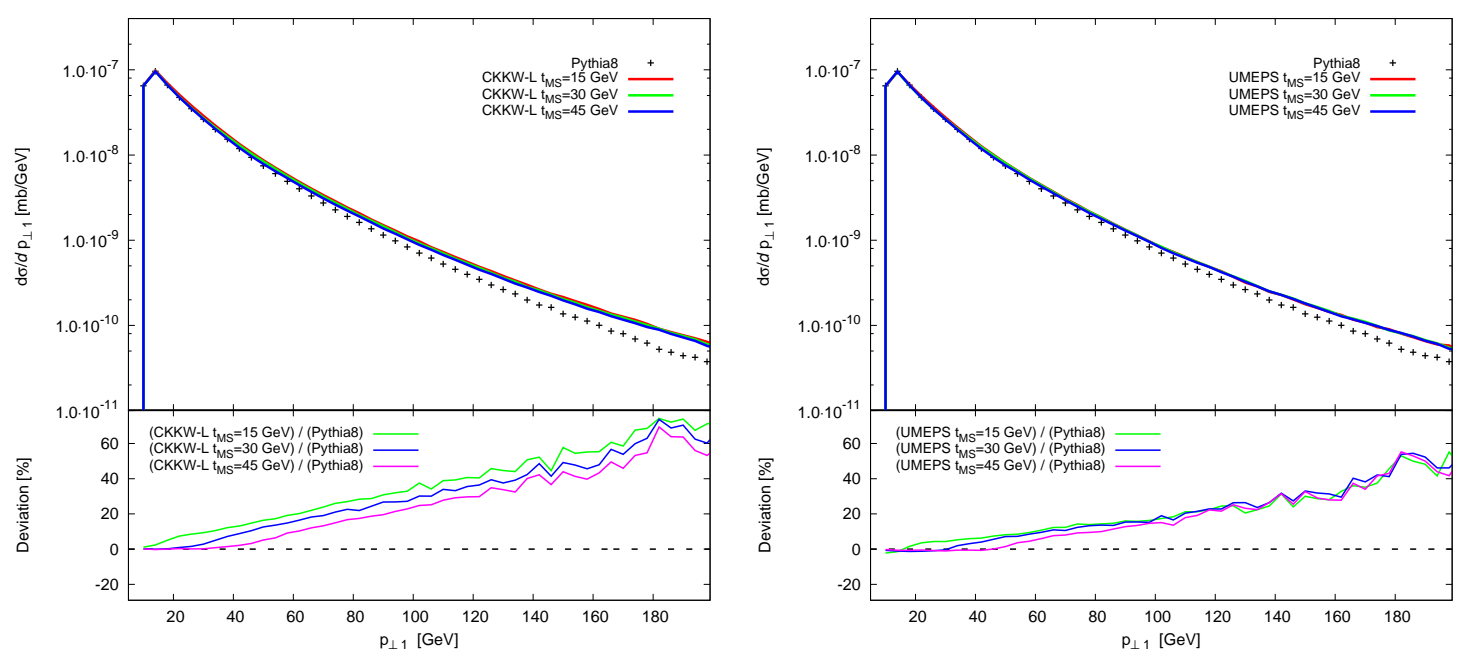

Figure 4: Transverse momentum of the hardest jet, for W-boson production in pp collisions at $E_{\mathrm{CM}}=7000 \mathrm{GeV}$, when merging up to two additional partons. Jets were defined with the $k_{\perp^{-}}$ algorithm, with $k_{\perp, \text { min }}=10 \mathrm{GeV}$. Multi-parton interactions and hadronisation were excluded. The lower insets show the deviation of merged results from default PYTHIA8, for three different $\rho_{\mathrm{MS}}$-values. Left panel: Results of the CKKW-L scheme. Right panel: Results of the UMEPS scheme.

has a significantly lower merging scale variation since the method enforces a cancellation of resolved and unresolved contributions.

Figure 5 shows that for very exclusive observables, CKKW-L and UMEPS are virtually indistinguishable. In this example, this is of course expected since the treatment of the highest multiplicity (here, the two-jet) matrix element is identical for both cases.

We would now like to perform a stress-test of the merging scale dependence. Since UMEPS properly cancels the effects of adding multi-jet matrix elements by subtracting their integrated counter-parts, it is in principle possible to push the merging scale to very small values. The variation of the inclusive cross section is shown in Figure 6. It is clear that UMEPS does indeed preserve the inclusive cross section, while for CKKW-L, very small merging scales lead to large changes, rendering the method unreliable. However, the error convergence in UMEPS is, due to the negative weights, significantly slower. We will comment on this below.

These unitarity violations might not induce drastic effects in the description of hardscale observables like the transverse-momentum distribution of the W-boson. However, magnifying the low-scale description of this observable (Figure 7) reveals problems. Figure 7 serves two purposes. It clearly shows that by pushing the merging scale to small values in CKKW-L, sub-leading contributions in the multi-jet matrix elements start to contribute more. Since those sub-leading contributions cannot be cancelled by the default parton shower, major increases over PYTHIA8 are found. UMEPS explicitly cancels these sub- 

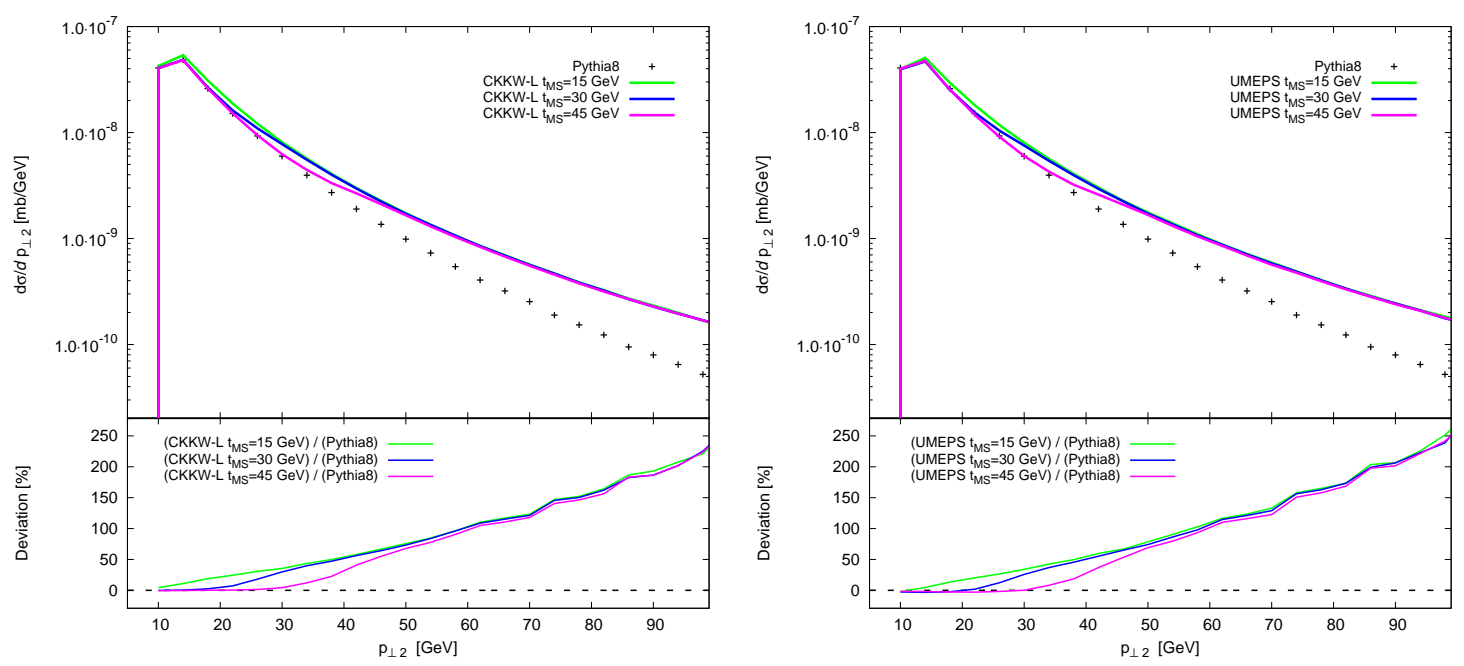

Figure 5: Transverse momentum of the second-hardest jet, for $\mathrm{W}$-boson production in pp collisions at $E_{\mathrm{CM}}=7000 \mathrm{GeV}$. Jets were defined with the $k_{\perp}$-algorithm, with $k_{\perp, \min }=10 \mathrm{GeV}$, when merging up to two additional partons. Multi-parton interactions and hadronisation were excluded. The lower insets show the deviation of merged results from default PYTHIA8, for three different $\rho_{\mathrm{MS} \text {-values. }}$ Left panel: Results of the CKKW-L scheme. Right panel: Results of the UMEPS scheme.

leading terms, and thus leads to a reliable prediction. The second observation in Figure 0 is the dependence on the primordial transverse momentum parameter $k_{\perp, p}$. This parameter was introduced in event generators to account for the transverse momentum of partons in the incoming protons, which cannot be generated in initial state DGLAP evolution. If this were the only effect to be modelled by $k_{\perp, p}$, a value of $k_{\perp, p} \approx 0.3 \mathrm{GeV}$ per incoming parton would seem appropriate. However, in current event generator tunes, significantly higher values $\left(k_{\perp, p} \approx 2 \mathrm{GeV}\right)$ are required [20,27], potentially to compensate for an incomplete phase space coverage in initial state showers due to the shower cut-off. The value of $k_{\perp, p}$ is mainly fixed by tuning to the position of the peak of the transverse momentum spectrum of the Z- or $\mathrm{W}$ boson. Increasing the value of $k_{\perp, p}$ roughly corresponds to pushing the peak to higher $p_{\perp}$ values. Figure 7 compares the UMEPS and CKKW-L predictions for the transverse momentum of the $\mathrm{W}$ boson, with $k_{\perp, p}=0.5 \mathrm{GeV}$, to default, tuned PYTHIA8 with $k_{\perp, p}=2.0 \mathrm{GeV}$. Unitarity violations in CKKW-L pull the peak back to lower $p_{\perp}$. This fact is virtually unchanged if we had used $k_{\perp, p}=2.0$ for CKKW-L predictions instead, suggesting that if we positively wanted to use a very low merging scale, an increase in $k_{\perp, p}$ would be necessary. UMEPS on the other hand can be used with very low merging scales, and in particular shows the interesting feature of matching the default PYTHIA8 curve without having a high $k_{\perp, p}$ value. We believe this is due to a better modelling of logarithms of the form $\ln (1 / x)$, which are present in the matrix element, and which are included in a unitary way in UMEPS - allowing for a much more natural value of $k_{\perp, p}$. 


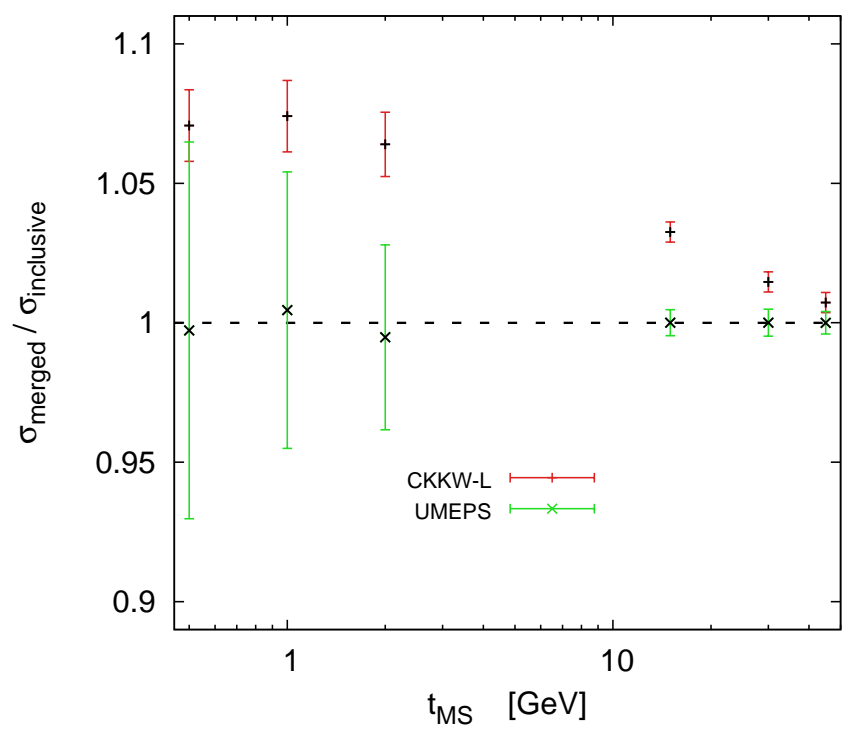

Figure 6: Inclusive cross section for UMEPS- and CKKW-L merging of up to two additional jets in W-boson production in pp collisions at $E_{\mathrm{CM}}=7000 \mathrm{GeV}$ (labelled $\sigma_{\text {merged }}$ ), in comparison to the the lowest-multiplicity inclusive cross section $\sigma_{\text {inclusive. The }}$ error bars represent only the statistical error on the merged cross section. For the UMEPS sample this becomes large for small merging scales, but as it is the same samples which are added and the subtracted, the central value stays very close to unity.

This result is of course very preliminary, since there are e.g. correlations of the shower cut-off $p_{\perp \text { min }}$ and $k_{\perp, p}$. One would hope that matrix-element merging would allow to lower $p_{\perp \text { min }}$, which might mean having to make a compromise for the value of $k_{\perp, p}$. We will come back to these aspects when presenting tunes for matrix-element-merged PYTHIA8 in a future publication.

\subsection{Dijet production}

We would further like to mention QCD dijet production at the LHC, since potential merging scale dependencies enter already when merging dijet- and three-jet matrix elements, and to demonstrate the flexibility of our implementation. The main objective of including QCD dijet production in this publication was to assess the treatment of MPI discussed at the end of section 4.1. This is most effectively done by comparing to data, and before these we would like to only stress one issue.

Figure 8 shows the transverse momentum of the third-hardest jet. We see that UMEPS and CKKW-L show very similar changes when compared to PYTHIA8. However, neither curves show the high- $p_{\perp}$ increase seen in [16]. This is simply because we have revised the choice of the renormalisation scale in the core $2 \rightarrow 2$ QCD scattering. In [16], the two powers of $\alpha_{\mathrm{s}}\left(\mu_{R}\right)$ in the core $2 \rightarrow 2$ process were never touched, and thus were evaluated with the rather unfortunate choice $\mu_{R}=\mathrm{M}_{\mathrm{Z}}^{2}$ in the input Les Houches events. This has been rectified in the current release of (CKKW-L in) PYTHIA8, i.e. the scale choice 

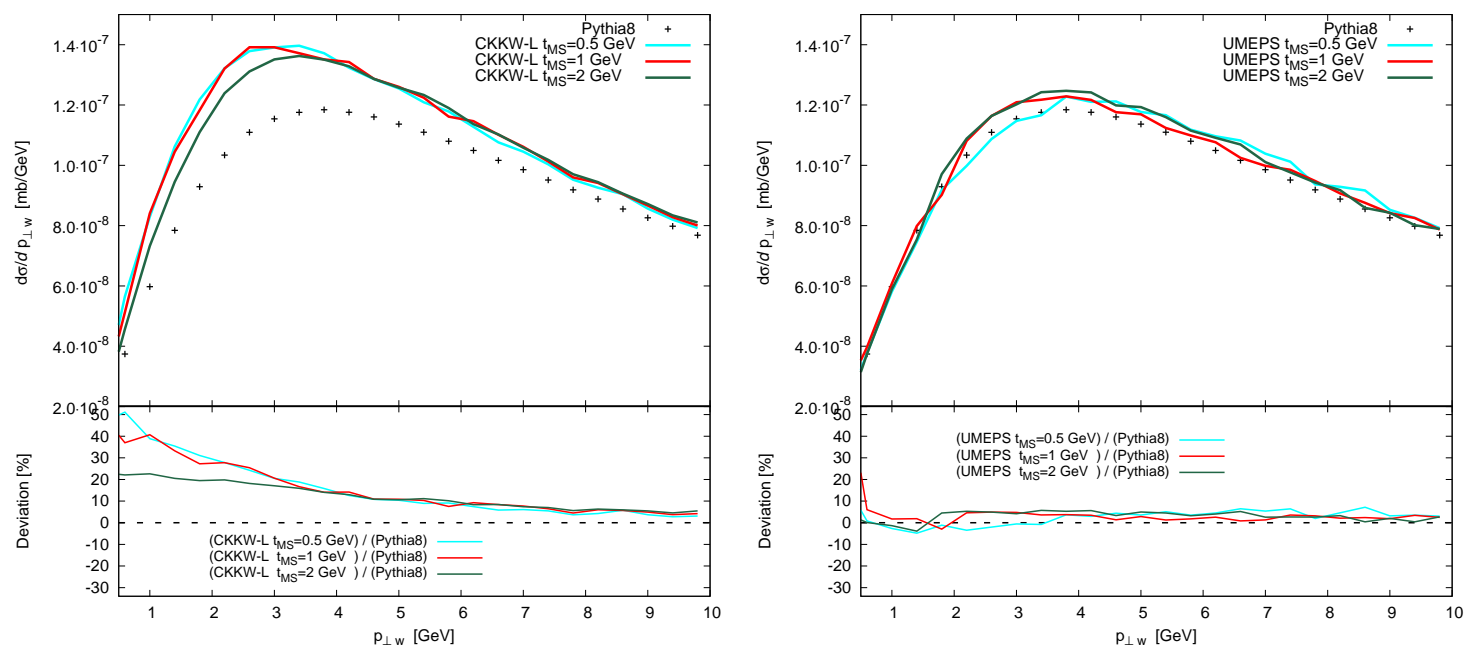

Figure 7: Transverse momentum of the W-boson, for W-boson production in pp collisions at $E_{\mathrm{CM}}=7000 \mathrm{GeV}$, when merging up to two additional partons. Multi-parton interactions and hadronisation were excluded. The PYTHIA8 results are generated with the default settings, in particular with a primordial transverse momentum of $k_{\perp, p}=2 \mathrm{GeV}$. All merged curves have been generated with $k_{\perp, p}=0.5 \mathrm{GeV}$. The lower insets show the deviation of merged results from default PYTHIA8, for three different $\rho_{\mathrm{MS}}$-values. Left panel: Results of the CKKW-L scheme. Right panel: Results of the UMEPS scheme.

$\left(\mu_{r, 2 \rightarrow 2}=m_{\perp, 1} m_{\perp, 1}\right)$ is now dynamical. Potential reweighting (due to the usage of fixed $\mu_{R}$ in the LHEF generation is handled internally in PYTHIA8. The trend that pure QCD multi-jet matrix elements have a softer spectrum of well-separated jets has already been observed in $[16,28]$. The merging scale variation in UMEPS is within the statistical error of the samples. The statical uncertainty is larger in UMEPS than in CKKW-L, due to cancellations between positive and negative weights (see the last part on section 6).

\subsection{Comparison with data}

In this section, we would like to confront UMEPS with experimental data. Event generator predictions were obtained with the settings of Tune A2 [29]. The results should of course not be regarded as final statement, since changes in the perturbative physics in event generators in principle request a full re-tuning. The intention of this section is to investigate if after including matrix-element information, hard-scale features are closer to measurements, and to assess the changes in underlying event description. All plots were produced with RIVET [30]. We apologise if the selection of experimental measurements seems biased.

In Figure 9, we show jet rates in W-boson production at the ATLAS [31]. We find an improved description after including up to two additional jets, and little differences between UMEPS and CKKW-L. 

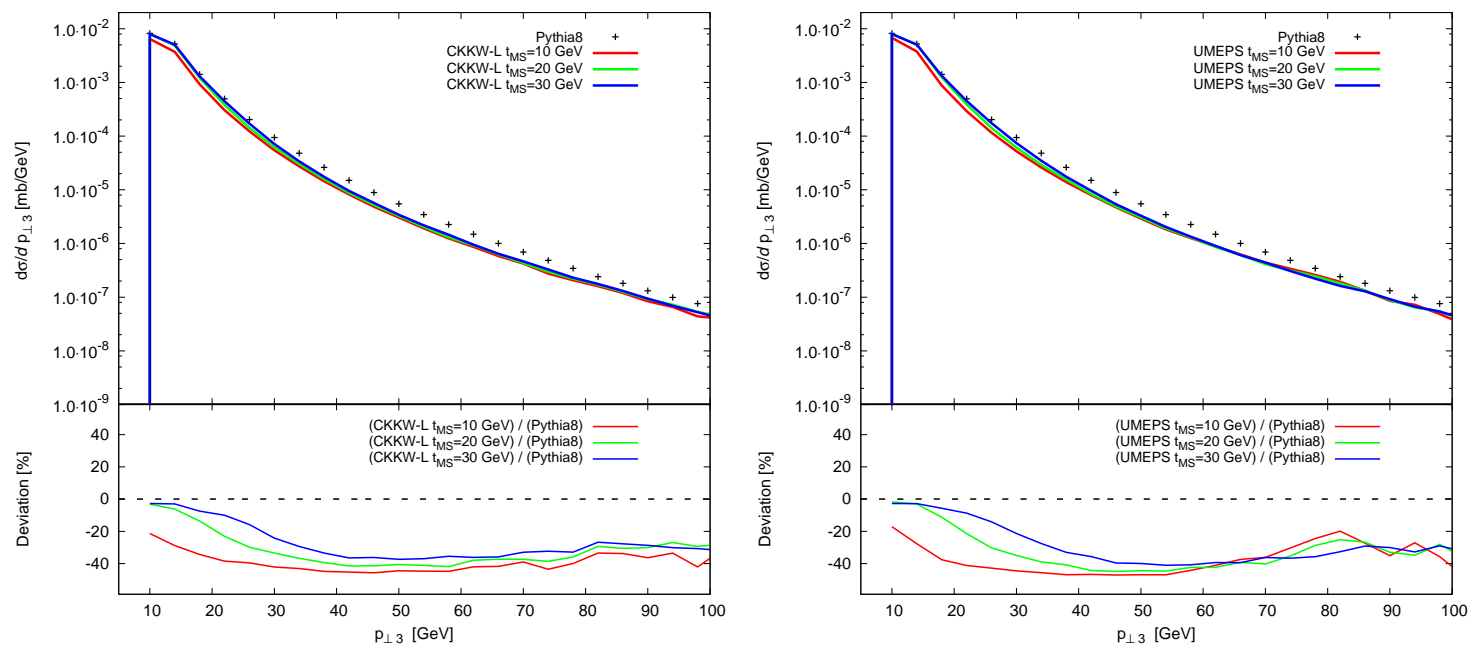

Figure 8: Transverse momentum of the third hardest jet, for pure QCD dijet production in pp collisions at $E_{\mathrm{CM}}=7000 \mathrm{GeV}$, when merging up to two additional partons. Jets were defined with the $k_{\perp}$-algorithm, with $k_{\perp, \text { min }}=10 \mathrm{GeV}$. Multi-parton interactions and hadronisation were excluded. The lower insets show the deviation of merged results from default PYTHIA8, for three different $\rho_{\mathrm{MS}}$-values. Left panel: Results of the CKKW-L scheme. Right panel: Results of the UMEPS scheme.
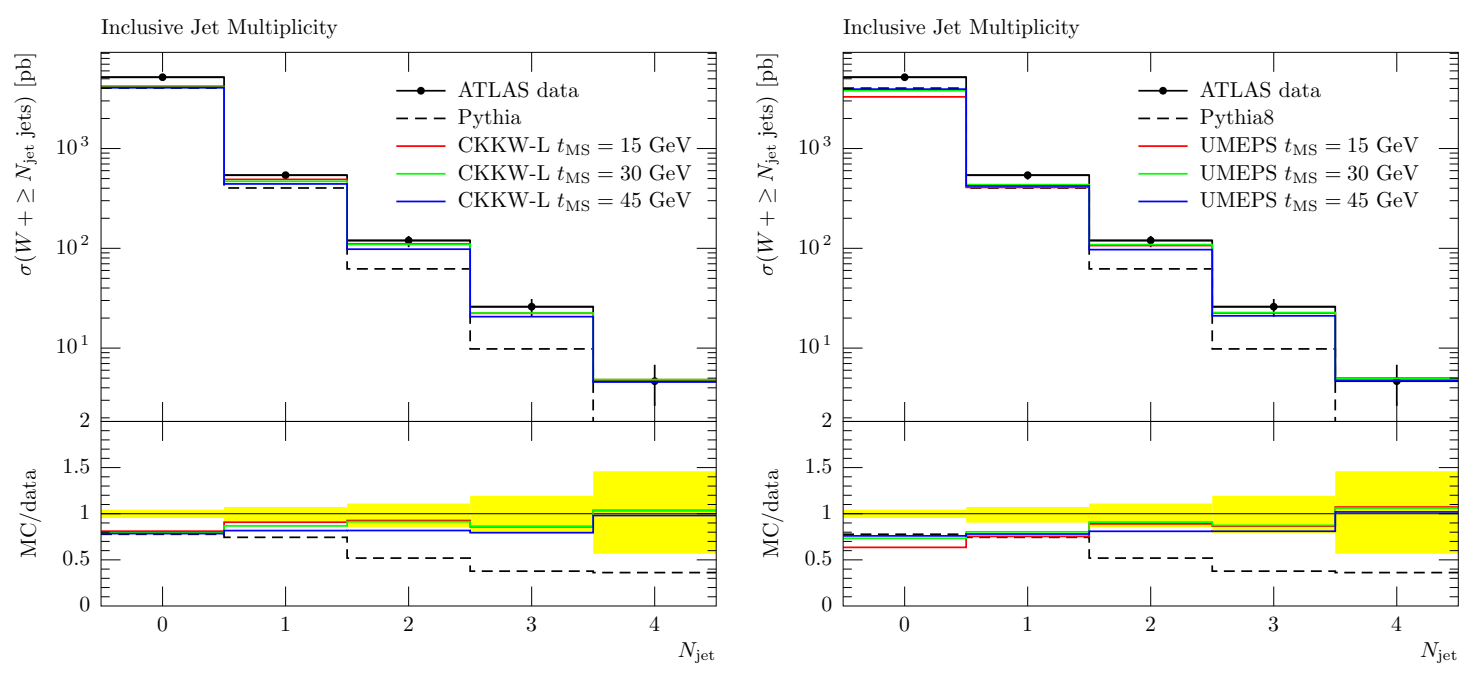

Figure 9: Jet multiplicity in W-boson production, for three different merging scales, as measured by ATLAS [31]. Effects of multiple scatterings and hadronisation are included.

The transverse momentum of the hardest jet is shown in Figure 10. Again, we find that the shape description is improved by the CKKW-L and UMEPS methods. CKKW-L shows merging scale variations at lower $p_{\perp}$-values, since a slightly different inclusive cross 

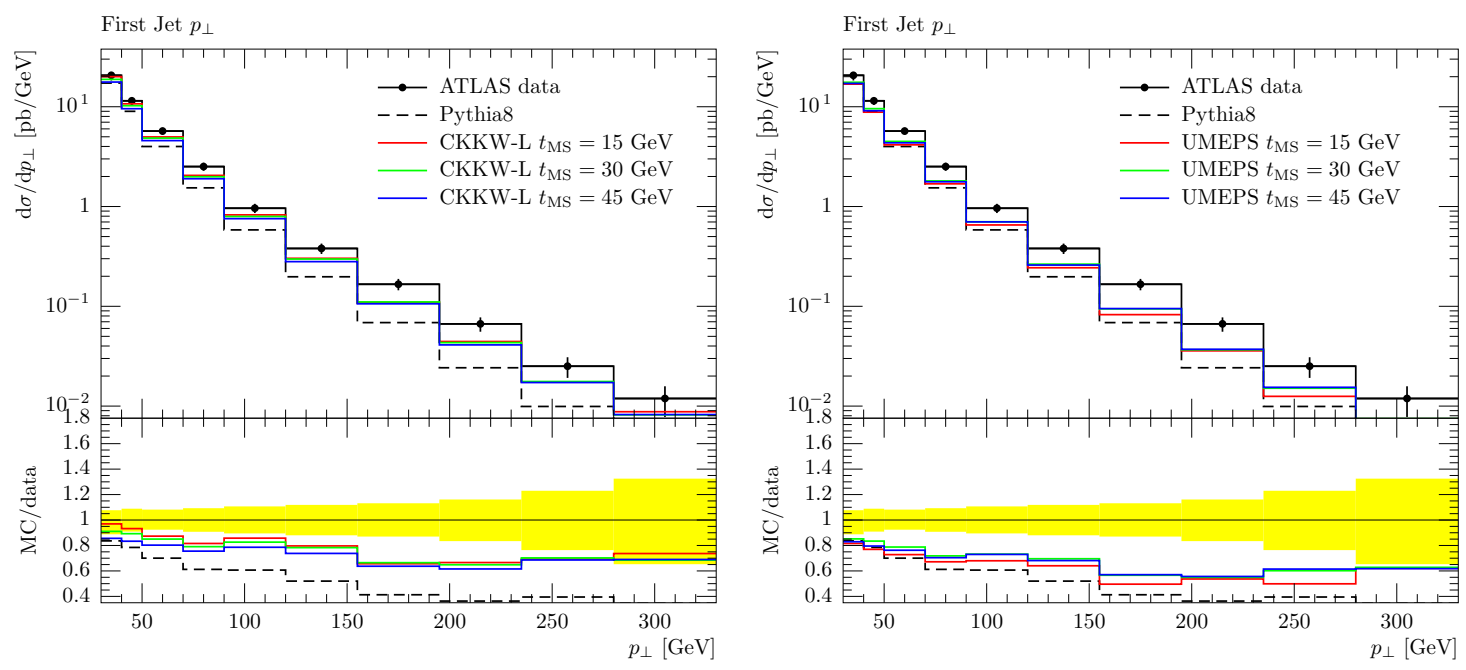

Figure 10: Transverse momentum of the hardest jet in W-boson production, for three different merging scales, as measured by ATLAS [31]. Effects of multiple scatterings and hadronisation are included.

section for a low merging scale leads to a slightly different normalisations. UMEPS on the other hand suffers from statistical fluctuations for a low merging scale value $\left(\rho_{\mathrm{MS}}=15\right.$ $\mathrm{GeV}$ ), while the curves for $\rho_{\mathrm{MS}}=30 \mathrm{GeV}$ and $\rho_{\mathrm{MS}}=45$ completely overlap. Note that the $p_{\perp}$ spectrum of UMEPS is a little softer than CKKW-L, in accordance with Figure 6.
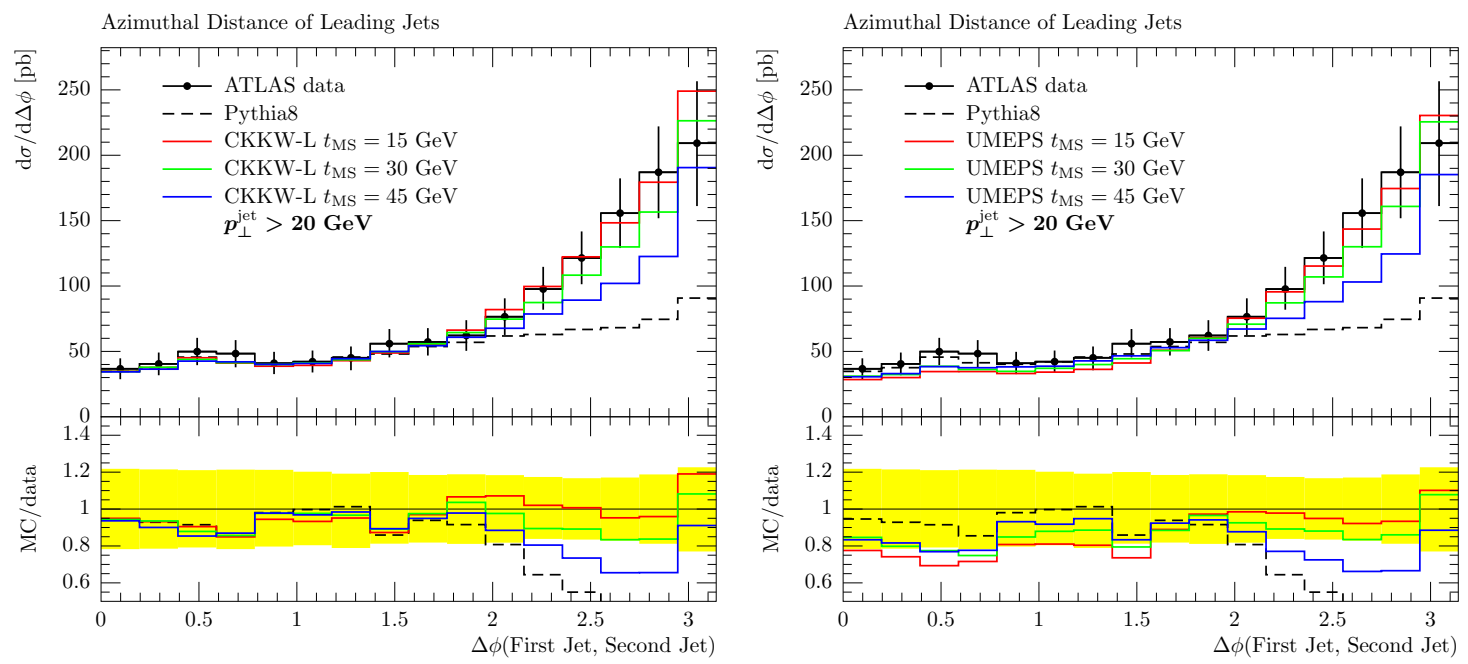

Figure 11: Azimuthal distance between the two hardest jets in W-boson production, for three different merging scales, as measured by ATLAS [31]. Effects of multiple scatterings and hadronisation are included.

It is interesting to investigate when tree-level matrix element merging schemes produce large uncertainties. Figure 11 shows the azimuthal distance $\Delta \phi_{12}$ between the two hardest 
jets. The parton shower alone cannot describe the peak at $\pi$. If the merging scale is low, the two-jet matrix element will give the dominant contribution in the peak region. High merging scales will increase the influence of the shower, thus degrading the description of the peak. Thus, this observable carries major merging scale variations, and provides excellent guidance for future improvements.
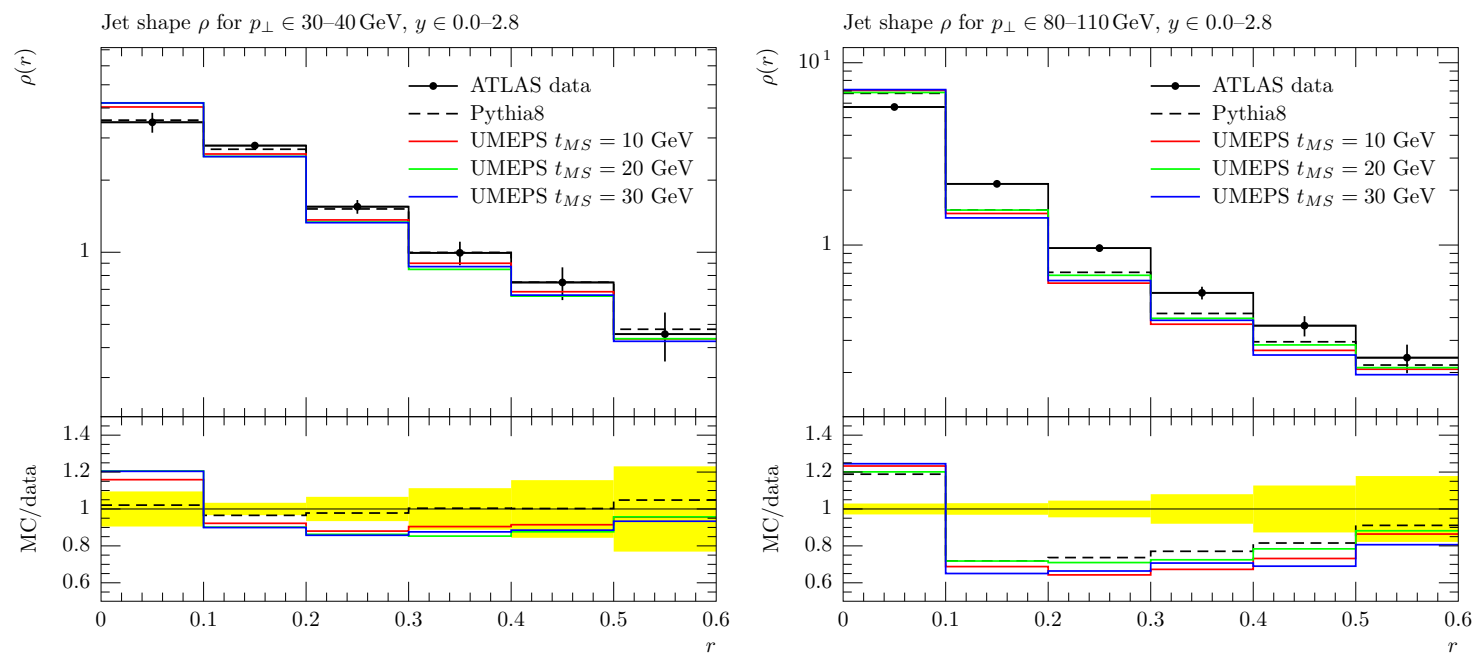

Figure 12: Jet shapes in QCD events, for three different merging scales, in two $p_{\perp}$ bins, as measured by ATLAS [32]. Effects of multiple scatterings and hadronisation are included.
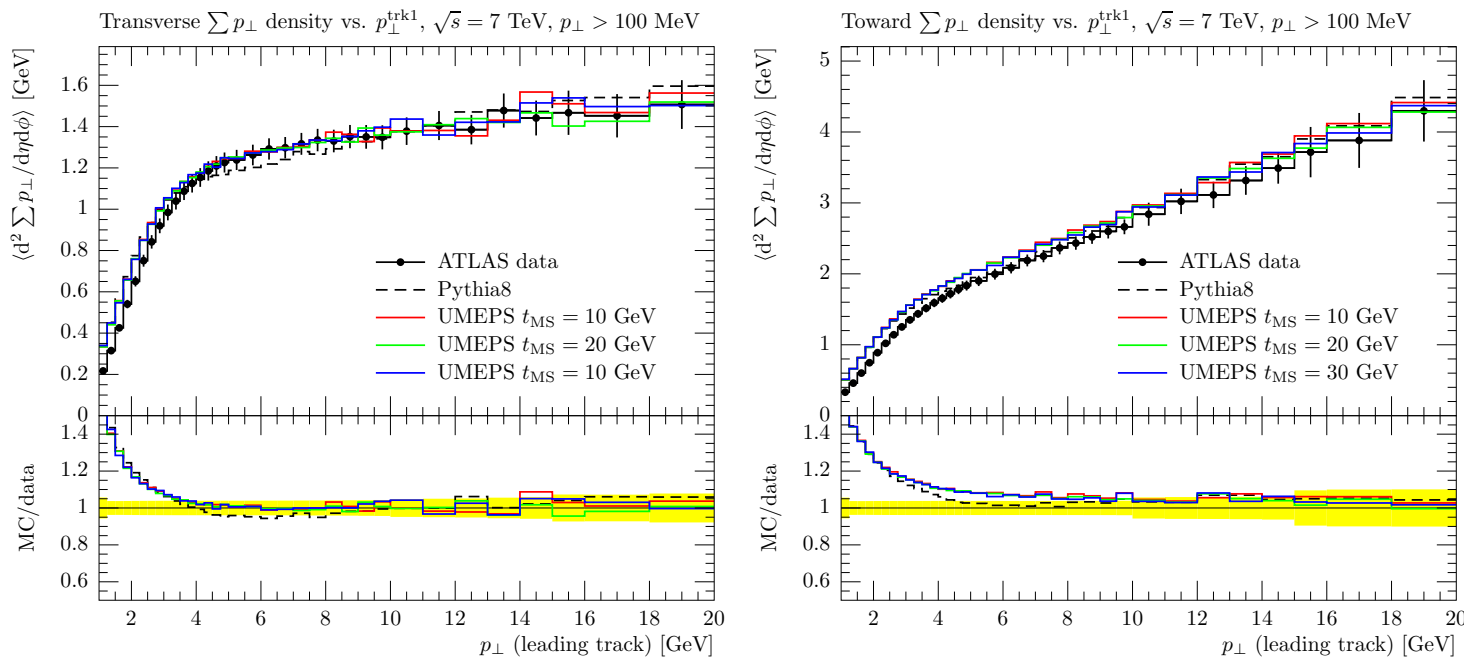

Figure 13: Sum of transverse momenta of charged particles in QCD events, for three different merging scales, in the transverse and toward region, as measured by ATLAS [33]. Effects of multiple scatterings and hadronisation are included.

Before moving to the discussion section, we would like to investigate how jet shapes at the LHC are changed by the inclusion of additional matrix elements in the pure QCD 
case. The ATLAS analysis [32] found that the differential jet shape for relatively low- $p_{\perp}$ jets with $p_{\perp} \leq 160 \mathrm{GeV}$ depends crucially on the modelling of the underlying event. In Figure 12, we show the default PYTHIA8 and UMEPS results for two $p_{\perp}$ bins. Although far from perfect, the difference between the pure shower and merged results are similar to what a minor change in $\alpha_{\mathrm{s}}$ would give ${ }^{6}$. We are confident that the prescription for adding MPI (see section 4.1) does indeed mean that the underlying-event modelling of PYTHIA8 is only marginally perturbed by the inclusion of additional jets. This is supported by Figure 13, which shows the sum of charged-particle transverse momenta in region close to the leading track (i.e. the toward region) and perpendicular to the leading track (transverse region) [33]. These are typical minimum-bias observables especially designed to investigate the underlying event, and PYTHIA8 tune A2 includes this data in the tuning procedure. It is reassuring that the inclusion of two additional jets through the UMEPS scheme did not invalidate this tuning.

\section{Discussion}

Before concluding this letter, we would like to make some comments to put this method into perspective.

\section{Relation to LoopSim}

Even though not completely obvious at first sight, UMEPS was heavily influenced by the LoopSim method [34]. This method as introduced to tame order-by-order large logarithmic enhancements by combining matrix elements with different jet multiplicities in a unitary way. The combination is done by joining all combinations to integrate over $1, \ldots, n$ partons in the ME event $S_{+n}$, and also allowing an integration over hard process particles. In figure 1, the final state gluons are candidates for integration (or, in the terminology of LoopSim, looping), and the $\mathrm{W}$-boson can be looped as well. With such a procedure, enhancements due to collinear $\mathrm{W}$-boson radiation off a dijet state can be compensated ${ }^{7}$.

Apart from major technical differences, one interesting difference is that in the LoopSim method, higher fixed-order corrections are approximated by multiple loopings, whereas in UMEPS, an all-order expression is included in the $S_{+n+1}$ state before one parton is looped. Integrating multiple emissions is only necessary for $\rho_{\mathrm{MS}}$ - unordered sequences of splittings, which are not considered in LoopSim. Furthermore, in UMEPS, only integrations of QCD splittings are performed, while LoopSim includes loopings of $\mathrm{W}$-boson radiation. We postpone the inclusion $\mathrm{W}$-boson clusterings in UMEPS until a full electroweak shower is available in PYTHIA8. It would clearly be interesting to combine the Sudakov resummation in UMEPS with the multiple loopings done in LoopSim. To arrive at a better description of $S_{+0}$ configurations, one could e.g. take $\alpha_{\mathrm{s}}^{1}$ contributions from the looped $S_{+1}$ state, $\alpha_{\mathrm{s}}^{2}$ contributions from the twice-looped $S_{+2}$, and all higher orders from Sudakov-reweighted

\footnotetext{
${ }^{6}$ This of course does not mean that we will to use this data for tuning, but that $\alpha_{\mathrm{s}}$-choices for different tunes can have a comparable effect on $\rho(r)$.

${ }^{7}$ At very large transverse momenta, the effect of multiple soft/collinear electroweak bosons becomes important. An appraisal of high- $p_{\perp}$ observables in WZ-production has recently been reported [35].
} 
thrice-integrated $S_{+3}$ events. The way the inclusive cross section is maintained in such a procedure will be less obvious than in the case of LoopSim or UMEPS.

\section{Merging scale dependence}

In the original CKKW-L algorithm it is evident that the dependence on the merging scale is absent to the accuracy of the PS. This means that for any observable, leading logarithmic terms on the form $\alpha_{\mathrm{S}}^{n} L^{2 n}$, where $L=\ln \mu_{F} / \rho_{\mathrm{MS}}$, are correctly cancelled to all orders. For a shower ${ }^{8}$ which in addition is correct to next-to-leading logarithmic accuracy, also terms on the form $\alpha_{\mathrm{s}}^{n} L^{2 n-1}$ are correctly cancelled.

What we have accomplished with the UMEPS method is that the total inclusive Bornlevel cross section is almost completely independent of the merging scale. In addition, if we look at the master formula in eq. (4.15), it is clear that for any phase space point $\phi_{n}$ with $n$ resolved partons, the inclusive cross section, integrating all contributions from higher parton multiplicities, is simply given by $\widehat{\mathrm{B}}_{n}$. Although $\widehat{\mathrm{B}}_{n}$ includes no-emission probabilities calculated by the shower, it has no dependence on the merging scale, and hence, all inclusive $n$-jet cross sections are independent of the merging scale. Since exclusive $n$-jet cross sections are the difference between the $n$-jet and $n+1$-jet inclusive cross sections, also these are independent of the merging scale.

The independence is, of course, not exact for any real observable. A jet algorithm will not cluster an $n+1$-jet state back to the precise $n$-parton phase space point as would the mapping of the parton shower, or symbolically for a general observable $\mathcal{O}$, $\int \mathcal{O}\left(S_{+n+1 j}\right) \widehat{\mathrm{B}}_{n+1} \neq \mathcal{O}\left(S_{+n j}\right) \int_{s} \widehat{\mathrm{B}}_{n+1 \rightarrow n}$. However, as long as the observable is collinearand infrared-safe this difference will not have any logarithmic enhancements, and as long as the $n$-jet state is well above the merging scale we can take this scale to be arbitrarily small, without changing the $n$-jet cross section.

Also, as we have noted before, there are some $n$-parton states, such as the one in figure 1, which do not have an underlying $n-1$-parton state reachable with a reconstructed PS emission. Unless the PS is amended with $W$-strahlung splittings, such contributions will always give a small dependence. However, we have found these to be numerically very small in the cases we have investigated.

\section{Events with negative weight}

Contrary to the standard CKKW(-L) algorithms our new UMEPS procedure will produce negatively weighted events. There has in the past been a great reluctance in the experimental community towards using generators with negative event weights. Mostly this has been a question about problems in handling the statistics and that it seems wasteful to spend a huge number of CPU cycles to do a full detector simulation on an event, which in the end will be cancelled by another event with a negative weight. However, the acceptance for negative weights have increased, and today most experiments are using programs such as MC@NLO [36-39], which do produce a fair amount of events with negative weight.

\footnotetext{
${ }^{8}$ Note that the shower in PYTHIA8 which is used in simulations in this paper has not been formally proven to be NLL-correct.
} 
Clearly UMEPS is more wasteful than CKKW-L in this respect, and the number of events that need to be analysed to get the same statistics is more than doubled. In fact it can be shown that the variance in the event weights, when calculating the noemission probability for the zero-jet case in CKKW-L with the Sudakov-veto algorithm, is proportional to $\Pi_{S_{+0}}-\Pi_{S_{+0}}^{2}$. The variance for UMEPS, where the corresponding factor is calculated by reclustering one-jet states multiplied by a no-emission probability, is of the form

$$
\frac{1-\Pi_{S_{+0}}}{-\ln \Pi_{S_{+0}}}-\left(\frac{1-\Pi_{S_{+0}}}{-\ln \Pi_{S_{+0}}}\right)^{2}
$$

Thus, for small merging scales (giving small no-emission probabilities), UMEPS becomes very inefficient as compared to CKKW-L.

We believe that the benefits of UMEPS outweigh this drawback. Also we note that the algorithm works in a way such that all events will either have zero weight or a weight of order $( \pm)$ unity. This is because the no-emission probabilities are generated by the Sudakovveto algorithm and are therefore either zero or unity, while the PDF- and $\alpha_{\mathrm{s}}$-reweighting typically is of $\mathcal{O}(1)$. Had the no-emission probabilities been calculated analytically, they would be very small for small merging scales, and e.g. each single 0-jet ME event would have to be cancelled by large number of small-weight reclustered 1-jet events. This would be very inefficient if a CPU-heavy detector simulation would have to be run on each event.

On the other hand, the Sudakov-veto algorithm causes some problem in the case full detector simulation is not used. In this case the computational bottle neck is typically in the ME generation of high jet multiplicities, and the problem is that most of these events are given zero weight and will be thrown away by the Sudakov-veto algorithm, especially for small merging scales. This can in principle be handled by a modification of the veto algorithm [40] where all events are kept but are given a small weight.

\section{Conclusions and Outlook}

In this article, we have presented a new method for tree-level matrix element merging called UMEPS. This method is heavily indebted to the CKKW-L prescription, but explicitly keeps the total inclusive cross section fixed. Since it builds on the implementation of CKKW-L in PYTHIA8, all developments of CKKW-L are immediately available to UMEPS. This for example includes improvements for BSM processes [41] and multiple pre-defined merging scales.

The UMEPS scheme uses an add-subtract prescription inspired by parton shower unitarity to combine the improved description of observable shapes of CKKW-L with a fixed total inclusive cross section. This means that significantly lower the merging scale values are possible, which allows for controlled improvement of low-scale features of the parton shower. Tuning efforts will be subject of a future article. When confronted with data, UMEPS and CKKW-L perform equally well.

UMEPS is an ideal candidate for further improvements, since the lowest-multiplicity cross section is not reweighted, making replacements with the full NLO or NNLO results 
possible. We have successfully implemented an NLO extension, and will present it in a separate publication [42].

Finally, while finishing this article, it came to our attention that a very similar approach has been developed in parallel by Plätzer [43], which further describes the extension of an inclusive-cross-section preserving merging method to NLO accuracy.

\section{Acknowledgements}

Work supported in part by the Swedish research council (contracts 621-2009-4076 and 6212010-3326). We would like to thank Simon Plätzer, Stefan Höche and Frank Tackmann for helpful discussions.

\section{References}

[1] N. Lavesson and L. Lönnblad, JHEP 12 (2008) 070, arXiv:0811.2912 [hep-ph].

[2] K. Hamilton and P. Nason, JHEP 1006 (2010) 039, arXiv:1004.1764 [hep-ph].

[3] S. Höche, F. Krauss, M. Schönherr, and F. Siegert, arXiv:1009.1127 [hep-ph].

[4] S. Alioli, K. Hamilton, and E. Re, JHEP 1109 (2011) 104, arXiv:1108.0909 [hep-ph].

[5] K. Hamilton, P. Nason, and G. Zanderighi, JHEP 1210 (2012) 155, arXiv:1206.3572 [hep-ph].

[6] T. Gehrmann, S. Höche, F. Krauss, M. Schönherr, and F. Siegert, arXiv:1207.5031 [hep-ph].

[7] S. Höche, F. Krauss, M. Schönherr, and F. Siegert, arXiv:1207.5030 [hep-ph].

[8] R. Frederix and S. Frixione, arXiv:1209.6215 [hep-ph].

[9] C. W. Bauer, F. J. Tackmann, and J. Thaler, JHEP 0812 (2008) 010, arXiv:0801.4026 [hep-ph].

[10] C. W. Bauer, F. J. Tackmann, and J. Thaler, JHEP 0812 (2008) 011, arXiv:0801.4028 [hep-ph].

[11] C. Bauer et al., (preprint in preparation). See eg. talk presented at the PSR12 workshop https://indico.desy.de/conferenceTimeTable.py?confId=5230\#20120530.

[12] S. Catani, F. Krauss, R. Kuhn, and B. R. Webber, JHEP 11 (2001) 063, arXiv:hep-ph/0109231.

[13] L. Lönnblad, JHEP 05 (2002) 046, arXiv:hep-ph/0112284.

[14] N. Lavesson and L. Lönnblad, JHEP 07 (2005) 054, arXiv:hep-ph/0503293.

[15] S. Höche, F. Krauss, S. Schumann, and F. Siegert, JHEP 05 (2009) 053, arXiv:0903.1219 [hep-ph].

[16] L. Lönnblad, and S. Prestel, JHEP 1203 (2012) 019, arXiv:1109.4829 [hep-ph].

[17] M. Bengtsson and T. Sjostrand, Nucl.Phys. B289 (1987) 810.

[18] M. Bengtsson and T. Sjostrand, Phys.Lett. B185 (1987) 435. 
[19] G. Miu, arXiv:hep-ph/9804317 [hep-ph].

[20] G. Miu and T. Sjostrand, Phys.Lett. B449 (1999) 313-320, arXiv:hep-ph/9812455 [hep-ph].

[21] W. T. Giele, D. A. Kosower, and P. Z. Skands, Phys.Rev. D78 (2008) 014026, arXiv:0707.3652 [hep-ph].

[22] W. Giele, D. Kosower, and P. Skands, Phys.Rev. D84 (2011) 054003, arXiv:1102.2126 [hep-ph].

[23] T. Sjöstrand, S. Mrenna, and P. Skands, Comput. Phys. Commun. 178 (2008) 852-867, arXiv:0710.3820 [hep-ph].

[24] P. Nason, JHEP 11 (2004) 040, arXiv: hep-ph/0409146.

[25] J. Alwall et al., Comput. Phys. Commun. 176 (2007) 300-304, hep-ph/0609017.

[26] M. Cacciari, G. P. Salam, and G. Soyez, Eur.Phys.J. C72 (2012) 1896, arXiv:1111.6097 [hep-ph].

[27] C. Balazs, J. Huston, and I. Puljak, Phys.Rev. D63 (2001) 014021, arXiv:hep-ph/0002032 [hep-ph].

[28] R. Corke and T. Sjöstrand, JHEP 03 (2011) 032, arXiv:1011.1759 [hep-ph].

[29] T. A. collaboration.

[30] A. Buckley et al., arXiv:1003.0694 [hep-ph].

[31] ATLAS Collaboration Collaboration, G. Aad et al., Phys.Rev. D85 (2012) 092002, arXiv:1201.1276 [hep-ex].

[32] Atlas Collaboration Collaboration, G. Aad et al., Phys.Rev. D83 (2011) 052003, arXiv:1101.0070 [hep-ex].

[33] Atlas Collaboration Collaboration, G. Aad et al., Phys.Rev. D83 (2011) 112001, arXiv:1012.0791 [hep-ex]].

[34] M. Rubin, G. P. Salam, and S. Sapeta, JHEP 1009 (2010) 084, arXiv:1006.2144 [hep-ph].

[35] F. Campanario and S. Sapeta, Phys.Lett. B718 (2012) 100-104, arXiv:1209.4595 [hep-ph].

[36] S. Frixione and B. R. Webber, arXiv:hep-ph/0612272.

[37] S. Höche, F. Krauss, M. Schönherr, and F. Siegert, JHEP 1209 (2012) 049, arXiv:1111.1220 [hep-ph].

[38] S. Höche, F. Krauss, M. Schönherr, and F. Siegert, Physical Review Letters (2012), arXiv:1201.5882 [hep-ph].

[39] V. Hirschi, R. Frederix, S. Frixione, M. V. Garzelli, F. Maltoni, et al., JHEP 1105 (2011) 044, arXiv:1103.0621 [hep-ph].

[40] L. Lönnblad, arXiv:1211.7204 [hep-ph].

[41] H. K. Dreiner, M. Kramer, and J. Tattersall, Europhys.Lett. 99 (2012) 61001, arXiv:1207.1613 [hep-ph].

[42] L. Lönnblad and S. Prestel, arXiv:1211.7278 [hep-ph].

[43] S. Plätzer, arXiv:1211.5467 [hep-ph]. 